\title{
Optimal sensor placement for multi-setup modal analysis of structures
}

\author{
Jie Zhang ${ }^{\mathrm{a}, *}$, Kristof Maes ${ }^{\mathrm{a}}$, Guido De Roeck ${ }^{\mathrm{a}}$, Edwin Reynders ${ }^{\mathrm{a}}$, Costas Papadimitriou ${ }^{\mathrm{b}}$, Geert Lombaert $^{\mathrm{a}}$ \\ ${ }^{a} K U$ Leuven, Department of Civil Engineering, Kasteelpark Arenberg 40, 3001 Leuven, Belgium \\ ${ }^{b}$ University of Thessaly, Department of Mechanical Engineering, Volos 38334, Greece
}

\begin{abstract}
Modal tests on large structures are often performed in multiple setups for practical reasons. Several sensors are kept fixed as reference sensors over all setups, while the other, so called roving sensors, are moved from one setup to another. This paper develops an optimal sensor placement strategy for multi-setup modal identification, which simultaneously optimizes the locations of the reference sensors and roving sensors. As an optimality criterion, the Information Entropy is adopted, which is a scalar measure of uncertainty in the Bayesian framework. The focus in the application goes to repetitive structures where modes typically occur in clusters, with closely spaced natural frequencies and similar wavelengths. The proposed strategy is illustrated for selecting optimal positions of uni-axial sensors for a repetitive frame structure. The influence of the number of reference sensors and two strategies for positioning roving sensors, i.e. a cluster and a uniform distribution of roving sensors, are investigated. The number of reference sensors is found to be preferably equal to or larger than the number of modes to be identified. In this case, the information content, as quantified by the Information Entropy, is not very sensitive to the roving sensor strategy. If less reference sensors are used, it is highly preferred to distribute the roving sensors uniformly over the structure instead of clustering them. The proposed strategy has been validated by an experimental modal test on a floor of an office building of KU Leuven, which has a nearly repetitive structural layout. The results show how optimally locating sensors allows extracting more information from the data. Though the focus is on applications involving repetitive structures, the proposed strategy can be applied to multi-setup modal identification of any large structure.
\end{abstract}

Keywords: multi-setup, modal identification, repetitive structures, mode clustering, optimal sensor placement

\section{Introduction}

Modal analysis [1, 2], i.e. identifying modal characteristics from measured responses, can be used for many purposes, such as model updating, structural health monitoring, damage detection and structural control. The accuracy of the identified modal characteristics depends on the number and locations of sensors. The number of positions covered in the experiments should be sufficiently large to accurately represent the mode shapes, i.e., to avoid spatial aliasing. Due to practical limitations on the number of sensors, modal testing of large structures is usually carried out in multiple setups [3]. The number of setups determines the measurement time. It is therefore necessary to find the optimal number and locations of the reference and roving sensors in order to obtain the required information within a reasonable time.

A careful choice of sensor positions is particularly important for repetitive structures, which have regular structural layout, e.g. buildings with repetitive floor plan. In such structures, modes with clustered natural frequencies occur [4]. It is important to determine proper sensor locations such that the closely spaced

\footnotetext{
*Corresponding author. Tel.: +3216325533

Email address: jie.zhang@kuleuven.be (Jie Zhang)
}

Postprint submitted to Journal of Sound and Vibration

Published version: J. Zhang, K. Maes, G. De Roeck, E. Reynders, C. Papadimitriou and G. Lombaert. Optimal sensor placement for multi-setup modal analysis of structures. Journal of Sound and Vibration, 401:214-232, 2017. https://doi.org/10.1016/j.jsv.2017.04.041 
modes are distinguished in modal identification. The distinction of modes is necessary, for example, in model calibration when pairing the identified and computed modes.

The dynamic behaviour of repetitive (periodic) structures has been investigated by means of wave propagation analysis $[5,6]$ based on the Floquet theory. Characteristic free waves and propagation constants are used to describe the dynamic behaviour of infinite periodic structures [7]. For a one-dimensional infinite periodic structure, $n_{\mathrm{c}}$ pairs of free waves can propagate through the structure at any frequency with $n_{\mathrm{c}}$ the number of coupling degrees of freedom (DOFs) between two adjacent units. Each pair contains identical but opposite going free waves, characterized by a negative and positive pair of propagation constants. The real part of the propagation constant represents the attenuation of the wave across the unit cell and the imaginary part represents the corresponding phase change.

The natural frequencies of finite periodic structures have been studied based on the wave propagation analysis for both mono-coupled [8] and multi-coupled [9] one dimensional periodic structures. For monocoupled structures, the adjacent units of a periodic structure are coupled by a single DOF; while in a multi-coupled structure, the units are connected by multiple DOFs. In [8], the natural frequencies of finite periodically supported beams, representing mono-coupled one-dimensional periodic structures with symmetric units, were studied by analyzing the propagation constants. Both simply supported and clamped ends were considered as the boundary conditions of the beams. It was found that the natural frequencies lie inside or at the boundaries of the propagation zones of the structure and the number of modes in each propagation zone is equal to the number of bays [8]. A propagation zone is a frequency range where the free characteristic wave is not decaying, i.e. the real part of the propagation constant is zero. It can therefore be concluded from the study in [8] that mode clustering occurs in the propagation zone of mono-coupled periodic structures with symmetric units and free or clamped boundaries. For multi-coupled periodic structures, the analysis becomes more involved as there is more than one pair of free waves at each frequency. In a frequency range where there is only a single pair of non-decaying waves, the number of modes is less than or equal to the number of bays if the periodic structure has a sufficiently large number of units [9]. The number of modes outside of these frequency ranges is difficult to predict on beforehand.

In order to distinguish between clustered modes in the modal identification of repetitive structures, a careful choice of sensor locations is needed. Optimal sensor placement has received considerable attention in the field of structural dynamics [10-19]. Criteria proposed for optimal sensor placement include the Modal Kinetic Energy [11, 12], Effective Independence [11], some norm (e.g. determinant and trace) of the Fisher Information Matrix [13, 14], Information Entropy (IE) [15] and the off-diagonal terms of the Modal Assurance Criterion (MAC) matrix [16]. Modal Kinetic Energy [11, 12] is used to select the sensor locations with possibly the largest modal responses. Effective Independence [11] aims at selecting the sensor locations such that the observed modes are linearly independent. It was found that the Effective Independence method is an iterated version of the Modal Kinetic Energy method [17]. The Effective Independence method is intrinsically equivalent to maximizing the determinant of the Fisher Information Matrix. The Fisher Information Matrix $[13,14]$ is the inverse of the covariance matrix of the estimates characterizing the uncertainty on the estimated parameters, which can be modal coordinates for modal identification or parameters related to the stiffness, mass and damping of the structure for parameter estimation. When the sensor locations are chosen to maximize some norm (determinant, trace) of the Fisher Information Matrix, the estimation uncertainty is minimized. In the Bayesian framework for parameter estimation, Papadimitriou, Beck and Au [15] have proposed to minimize the Information Entropy. The Information Entropy is defined as a scalar measure of the uncertainty in the parameter estimates. If the number of data is sufficiently large, minimizing the Information Entropy becomes equivalent to maximizing the determinant of the Fisher Information Matrix [18]. The influence of the spatial correlation of the prediction errors on the Information Entropy [19] was studied. It was found that this avoids closely spaced sensors which are generally believed to provide redundant information. The Modal Assurance Criterion (MAC) is a measure of the collinearity between two mode shape vectors $[1,20]$. Minimizing the off-diagonal terms of the MAC matrix results in less dependent mode shape vectors. The Modal Kinetic Energy method, Effective Independence method and the MAC matrix method can be used to determine the optimal sensor locations for modal identification of structures. The Fisher Information Matrix method and Information Entropy method are suitable for both modal identification and parameter estimation, depending on the type of the estimation parameters. 
Finding the optimal sensor locations by full enumeration of all sensor configuration candidates is difficult or even impossible when the number of possible sensor locations is large. A number of studies have focused on computational algorithms which can improve the efficiency, including heuristic algorithms [18, 21, 22], genetic algorithms [23, 24] and meta-heuristic algorithms [25]. Genetic algorithms [23, 24] have been used to find optimal solutions of sensors locations. Studies on meta-heuristic algorithms inspired from nature were also conducted [25]. As an alternative to these algorithms which involve a random search component, Sequential Sensor Placement algorithms have been studied. Based on the Information Entropy, two heuristic algorithms i.e. Forward and Backward Sequential Sensor Placement (FSSP and BSSP) have been proposed [18] to find suboptimal sensor locations. It was found that these Sequential Sensor Placement algorithms generally provide a good approximation of the optimal sensor configuration at a very reasonable computational cost [19]. A heuristic sequential algorithm was proposed in [21] for selecting the optimal locations of various types of sensors base on the robust Information Entropy, which allows the evaluation of the overall performance of multiple types of sensors. Based on convex optimization, a heuristic method was proposed which selects suboptimal sensor locations and gives a performance bound [22]. An iterative algorithm based on Effective Independence method was also proposed to reduce the computational effort [11]. These studies mainly focused on optimal sensor placement for single setup measurements. Up to now, the problem of determining optimal positions of reference and roving sensors in a multi-setup modal identification has received much less attention.

An important step in multi-setup modal analysis is the data merging. The approaches for data merging fall into two categories depending on whether the merging is performed before or after modal identification. In Experimental Modal Analysis (EMA), the data from different setups are usually merged before identifying the modal characteristics [3]. The data in different setups are usually inconsistent, however, resulting in the identification of multiple spurious modes instead of a single mode near a natural frequency [26]. The classical approach for data merging in multi-setup Operational Modal Analysis (OMA) is the Post Separate Estimation Re-scaling (PoSER) approach [3]. The partial modes shapes in different setups are identified first and merged next to obtain the global mode shapes. The natural frequencies and damping ratios are averaged. This merging strategy can also be used in Experimental Modal Analysis to solve the inconsistency problem. However, problems may also arise in the PoSER approach: some modes may not be identified in some setups and it is difficult to pair modal characteristics of closely spaced modes. This will cause problems for multi-setup modal analysis of repetitive structures, which typically have closely spaced modes. Other data merging approaches have been investigated for Operational Modal Analysis, such as Post Global Estimation Re-scaling (PoGER) and Pre Global Estimation Re-scaling (PreGER), merging data after and before modal identification, respectively [3, 27].

In this contribution, a strategy of optimal sensor placement is proposed for multi-setup modal testing of large structures, focusing on repetitive structures. The outline of the paper is as follows. First, the mode clustering phenomenon and the similarity of the corresponding mode shapes are illustrated for the case of a periodic three dimensional frame structure to motivate the study. The relation between the propagation zones of the infinite periodic structure and the mode clustering frequency ranges is discussed. Next, a strategy for multi-setup modal testing is proposed where the aim is to select the sensor positions such that the problems for data merging after modal identification are avoided. This method is applicable for any large structure where modal testing is performed in multiple setups. The developed strategy is then illustrated for a three dimensional repetitive frame structure using uni-axial sensors. The influence of the number of reference sensors and two strategies for positioning roving sensors are studied. An experiment was performed on the office building "Blok D" of KU Leuven for validation of the proposed strategy.

\section{Wave propagation and mode clustering in repetitive structures}

For a mono-coupled periodic structure with symmetric units and free or clamped boundaries, mode clustering occurs in the propagation zones [8]. The relation between the propagation zones and mode clustering frequency is less clear for multi-coupled periodic structures, however. In this section, the mode clustering and wave propagation characteristics are investigated for a multi-coupled periodic frame structure. 
This is important for the physical understanding of the modal identification problems that arise in finite periodic structures.

A concrete frame structure with regular floor plan, shown in figure 1, is used to illustrate the wave propagation characteristics and phenomenon of mode clustering in the frequency range between $0 \mathrm{~Hz}$ and $50 \mathrm{~Hz}$. The floor of one module has a dimension of $5 \mathrm{~m} \times 5 \mathrm{~m}$ with a thickness of $0.15 \mathrm{~m}$. The story height is $3 \mathrm{~m}$. The beams in the transverse $(\mathrm{y})$ and longitudinal $(\mathrm{x})$ direction have a rectangular section of $0.2 \mathrm{~m} \times 0.5 \mathrm{~m}$. The columns have a square section of $0.35 \mathrm{~m} \times 0.35 \mathrm{~m}$. The concrete has a density of $\rho=2500 \mathrm{~kg} / \mathrm{m}^{3}$ and a Young's modulus of $E=35 \times 10^{9} \mathrm{~N} / \mathrm{m}^{2}$. Damping is not taken into account. In the following, the modal characteristics of this structure are studied as a function of the number of bays.

The repetitive frame structure is modeled in StaBIL [28], a finite element toolbox in Matlab. A four-noded shell element, which consists of a bilinear membrane element and four overlaid discrete Kirchhoff triangles (DKT) for bending, is used for the slab. The beams and columns are modeled using beam elements. The mesh size is determined by the smallest wavelength at $50 \mathrm{~Hz}$. The length of one element is limited to one eighth of the smallest wavelength, leading to a mesh of $12 \times 12$ elements for the plate of one bay, 12 elements for each beam and 6 elements for each column.

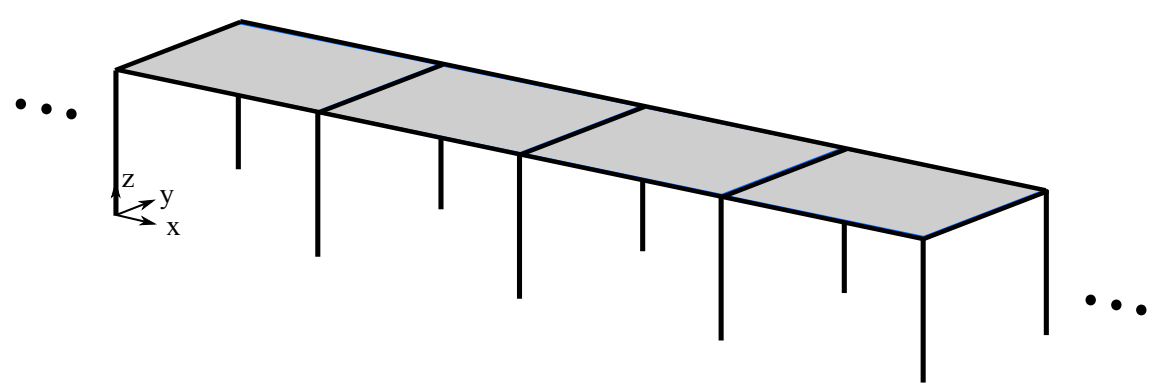

Figure 1: The repetitive frame structure with plates (light gray), columns and beams (black).

\subsection{Mode clustering in the finite repetitive frame structure}

The modal characteristics in the frequency range between $0 \mathrm{~Hz}$ and $50 \mathrm{~Hz}$ are calculated for the finite repetitive frame structure. The natural frequencies are shown in figure 2 as a function of the numbers of bays of the structure. Mode clustering is found to occur in the frequency bands with non-decaying free waves, as will be discussed in the next section.

The mode shapes in the frequency range between $14.0 \mathrm{~Hz}$ and $15.3 \mathrm{~Hz}$ corresponding to the first mode cluster are shown in figure 3 for the frame structure with twelve bays. The mode shapes show a pronounced bending of the floors. It can be seen that the modes are quite similar in some bays. For instance, the mode shapes of the two central bays are similar for modes 5, 7, 9, 11, 13 and 15 and modes 6, 8, 10, 12, 14 and 16, respectively. The MAC values $[1,20]$ between two mode shapes of the two central bays are calculated for modes $5,7,9,11,13$ and 15 and modes $6,8,10,12,14$ and 16, shown in figure 4. The MAC values of some modes are very high, indicating the similarity of the mode shapes. The mode shapes of the two central bays for modes 5 and 7 are nearly identical. 


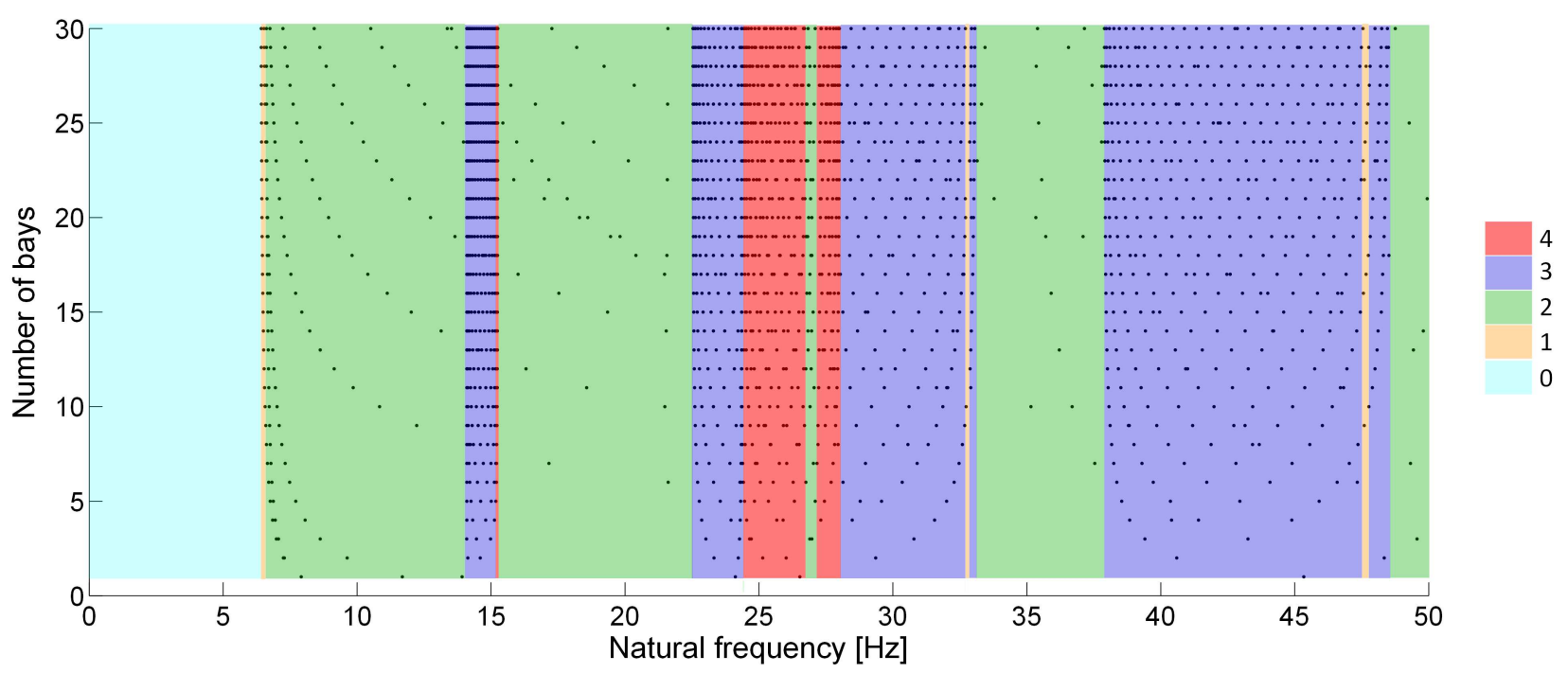

Figure 2: Natural frequencies as a function of the numbers of bays of the finite repetitive frame structure. The background color indicates the number of non-decaying free waves at each frequency, shown in the color bar.

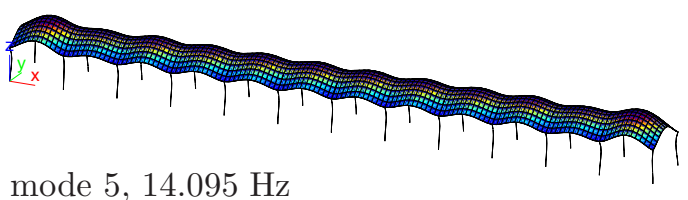

(a) mode 5, $14.095 \mathrm{~Hz}$

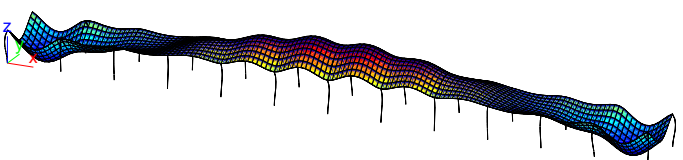

(c) mode $7,14.225 \mathrm{~Hz}$

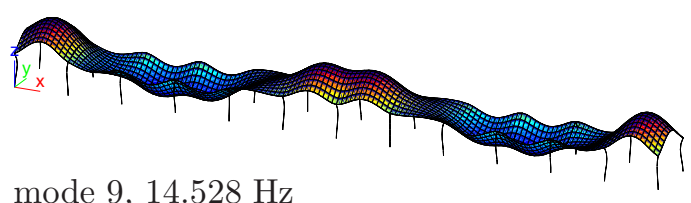

(e) mode $9,14.528 \mathrm{~Hz}$

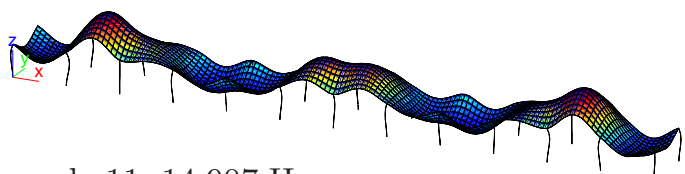

(g) mode 11, $14.907 \mathrm{~Hz}$

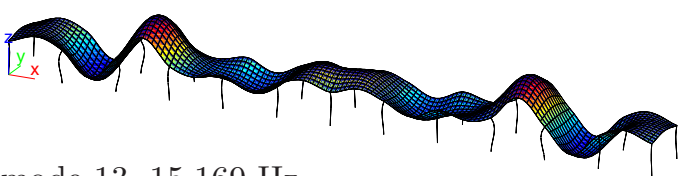

(i) mode 13, $15.169 \mathrm{~Hz}$

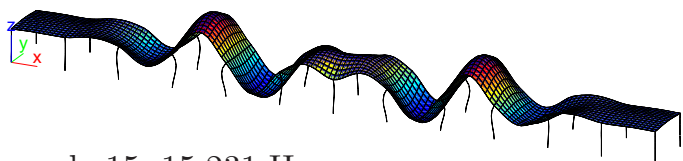

(k) mode 15, $15.231 \mathrm{~Hz}$

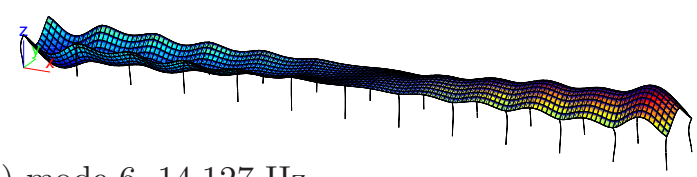

(b) mode 6, $14.127 \mathrm{~Hz}$

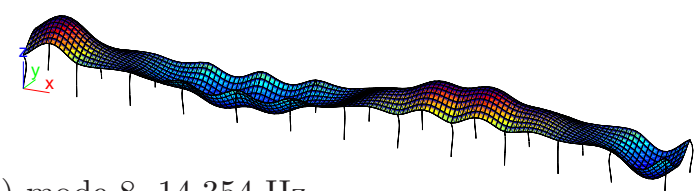

(d) mode 8, $14.354 \mathrm{~Hz}$

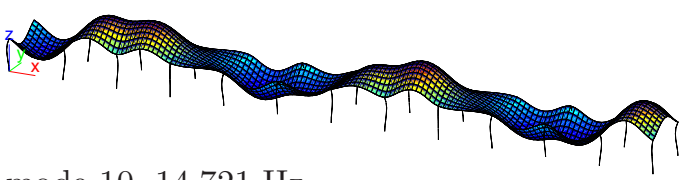

(f) mode 10, $14.721 \mathrm{~Hz}$

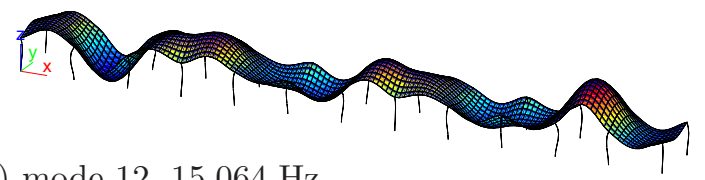

(h) mode 12, $15.064 \mathrm{~Hz}$

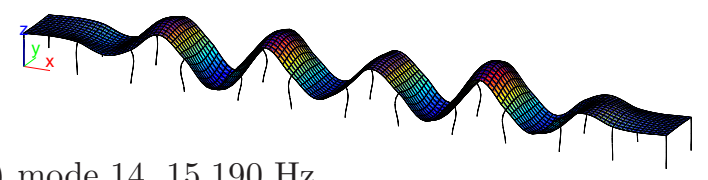

(j) mode 14, $15.190 \mathrm{~Hz}$

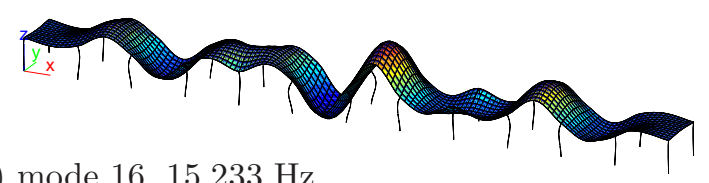

Figure 3: The modes of the twelve-bay frame in the first mode cluster (between $14.0 \mathrm{~Hz}$ and $15.3 \mathrm{~Hz}$ ). 


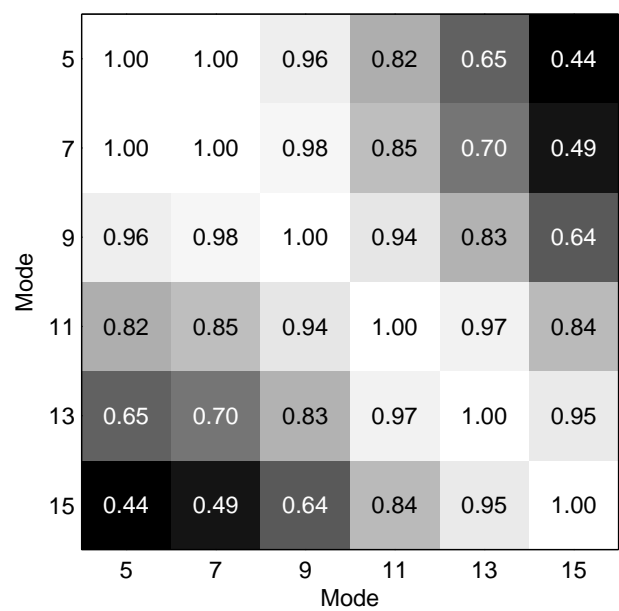

(a)

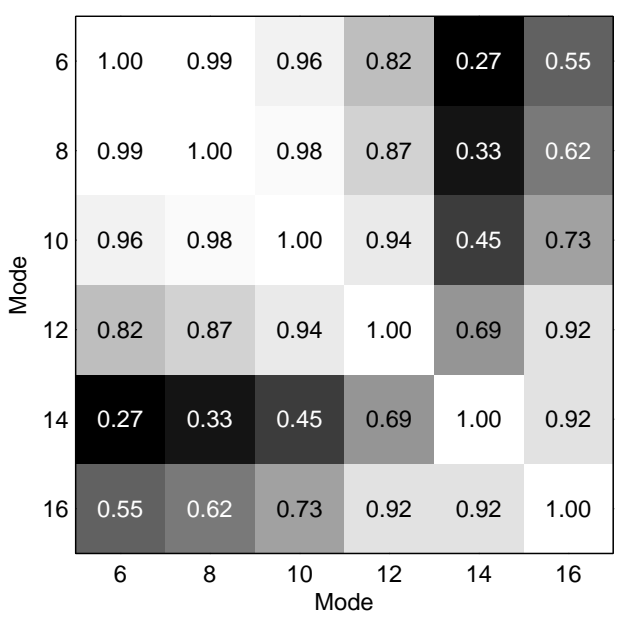

(b)

Figure 4: The MAC values for (a) modes 5, 7, 9, 11, 13 and 15 and (b) modes 6, 8, 10, 12,14 and 16 at the two central bays.

\subsection{Wave propagation in the infinite periodic frame structure}

The repetitive frame structure is regarded as a periodic structure with generalized boundary conditions (figure 5). The periodic unit consists of one plate, two columns, one beam in the transverse direction and two beams in the longitudinal direction. Generalized boundary conditions are introduced by the beam and columns at the right end of the structure.

The free waves which can propagate in the infinite periodic frame structure (figure 6) are calculated from the finite element model of a single periodic unit (figure 7) following the procedure in [29].

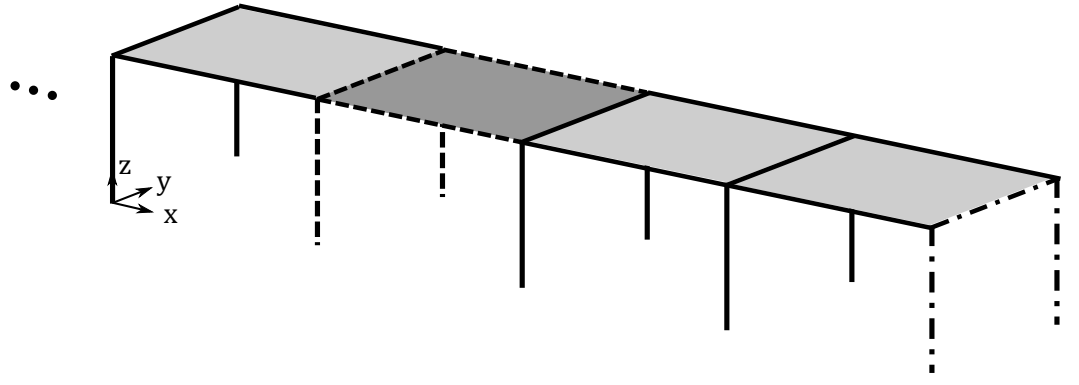

Figure 5: The finite repetitive frame structure consisting of a repetition of unit cells (dashed lines and dark gray area), followed by a generalized boundary (dot-dashed lines)

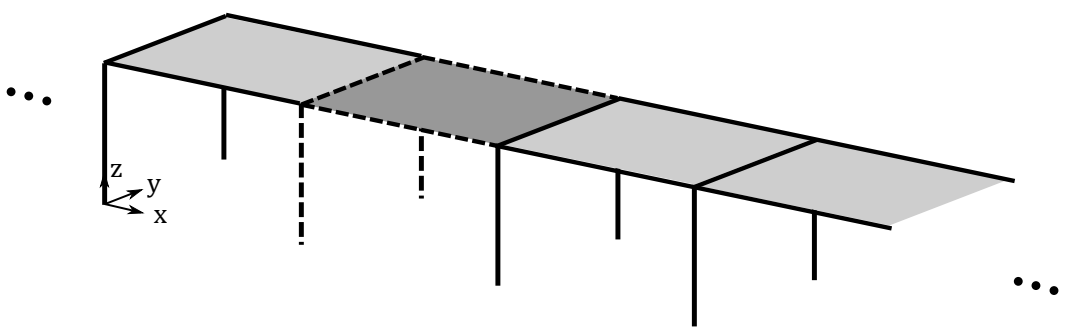

Figure 6: The infinite periodic frame structure. 


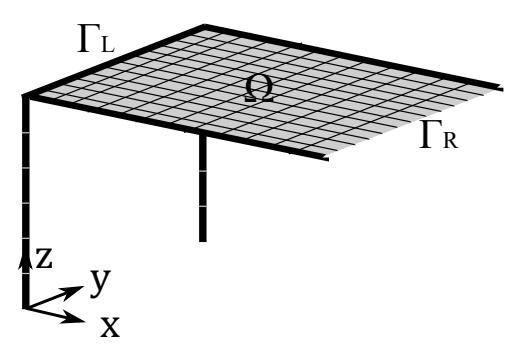

Figure 7: The mesh of a repetitive unit.

The DOFs of a repetitive unit are divided into two sets (figure 7 ): the interface DOFs $\left(\Gamma_{\mathrm{L}}\right.$ and $\left.\Gamma_{\mathrm{R}}\right)$ and the internal DOFs $(\Omega) . \Gamma_{\mathrm{L}}$ and $\Gamma_{\mathrm{R}}$ contain the DOFs that are coupled with the adjacent units on the left and right, respectively. $\Omega$ contains the remaining DOFs that are not coupled with the adjacent units.

After the elimination of the internal DOFs $\Omega$, the equation of motion of one unit, which gives the relation between the dynamic forces $\hat{\mathbf{F}}$ and displacements $\hat{\mathbf{u}}$ at the interface DOFs $\left(\Gamma_{\mathrm{L}}\right.$ and $\left.\Gamma_{\mathrm{R}}\right)$ of a unit, reads as follows:

$$
\left[\begin{array}{c}
\hat{\mathbf{F}}_{\mathrm{L}} \\
\hat{\mathbf{F}}_{\mathrm{R}}
\end{array}\right]=\left[\begin{array}{ll}
\mathbf{D}_{\mathrm{LL}} & \mathbf{D}_{\mathrm{LR}} \\
\mathbf{D}_{\mathrm{RL}} & \mathbf{D}_{\mathrm{RR}}
\end{array}\right]\left[\begin{array}{c}
\hat{\mathbf{u}}_{\mathrm{L}} \\
\hat{\mathbf{u}}_{\mathrm{R}}
\end{array}\right]
$$

where $\mathbf{D}$ is the dynamic stiffness matrix and the subscripts $\mathrm{L}$ and $\mathrm{R}$ represent the DOFs in $\Gamma_{\mathrm{L}}$ and $\Gamma_{\mathrm{R}}$, respectively.

The propagation constants $\mu$ and free waves $\boldsymbol{\psi}$ are found as the solution of the following eigenvalue problem [29]:

$$
\left[\begin{array}{cc}
\mathbf{D}_{\mathrm{RL}} & \mathbf{D}_{\mathrm{RR}} \\
\mathbf{0} & \mathbf{I}
\end{array}\right] \boldsymbol{\psi}=e^{\mu}\left[\begin{array}{cc}
-\mathbf{D}_{\mathrm{LL}} & -\mathbf{D}_{\mathrm{LR}} \\
\mathbf{I} & \mathbf{0}
\end{array}\right] \boldsymbol{\psi}
$$

Figure 7 shows the mesh of the periodic unit. There are seventy-eight $(13 \times 6)$ coupling DOFs at the interface, resulting in seventy-eight pairs of free waves at each frequency according to the Floquet theory. The propagation constants and free waves at $15 \mathrm{~Hz}$ are studied in order to gain a physical understanding of the waves propagating in the frame structure. There are three pairs of non-decaying waves at this frequency, the corresponding propagation constants are $\pm 0.12 i, \pm 0.62 i$ and $\pm 1.60 i$. The other seventy-five pairs are decaying waves.

A selection of free waves in the infinite periodic frame structure is plotted for a finite part of the infinite structure in figure 8. The real parts and imaginary parts of the three non-decaying free waves are shown in figure $8 \mathrm{a}-8 \mathrm{c}$. It can be seen that the three non-decaying free waves are a longitudinal wave, a lateral wave and a vertical bending wave. The wavelengths of the three types of waves are approximately $240 \mathrm{~m}, 50 \mathrm{~m}$ and $10 \mathrm{~m}$, respectively. Three selected decaying waves are plotted in figure 8d-8f. The decay rate depends on the real part of the propagation constant. After propagating through one unit, the amplitudes of the three decaying waves in figure $8 \mathrm{~d}-8 \mathrm{f}$ are reduced by $43 \%, 49 \%$ and $87 \%$, respectively.

The propagation constants are calculated in the frequency range between $0 \mathrm{~Hz}$ and $50 \mathrm{~Hz}$. The number of non-decaying waves is counted at each frequency. The frequency range is divided into zones according to the number of non-decaying waves, as indicated by the background color in figure 2. From figure 2, it is observed that the number of modes increases with the number of bays in the frequency range where non-decaying waves exist. The higher the number of non-decaying waves, the higher the modal density. For example, in the range between $14.0 \mathrm{~Hz}$ and $15.2 \mathrm{~Hz}$ where three non-decaying waves are found, the modal density is much higher than that in the range between $6.6 \mathrm{~Hz}$ and $14.0 \mathrm{~Hz}$ where two non-decaying waves are found. For this repetitive frame structure, mode clustering with a high modal density occurs in the frequency ranges with three or more non-decaying waves. The first mode cluster in the range between 14.0 $\mathrm{Hz}$ and $15.3 \mathrm{~Hz}$ has been considered previously, where three non-decaying waves are found between $14.0 \mathrm{~Hz}$ and $15.2 \mathrm{~Hz}$ and four non-decaying waves are found between $15.2 \mathrm{~Hz}$ and $15.3 \mathrm{~Hz}$. 


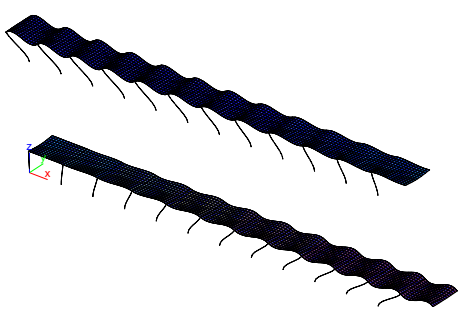

(a) $\mu= \pm 0.12 i$

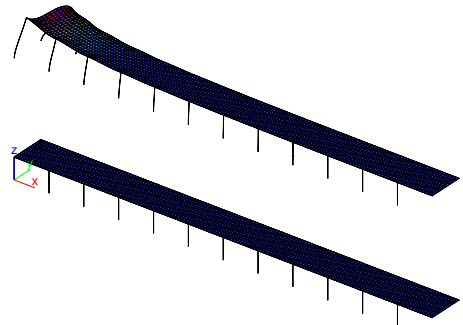

(d) $\mu= \pm 0.5698$

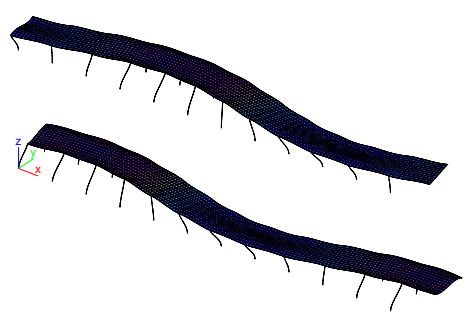

(b) $\mu= \pm 0.62 i$

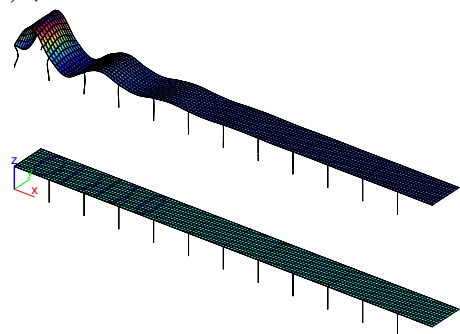

(e) $\mu= \pm 0.6676+3.1416 i$

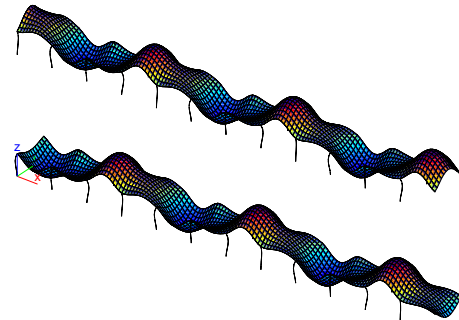

(c) $\mu= \pm 1.60 i$

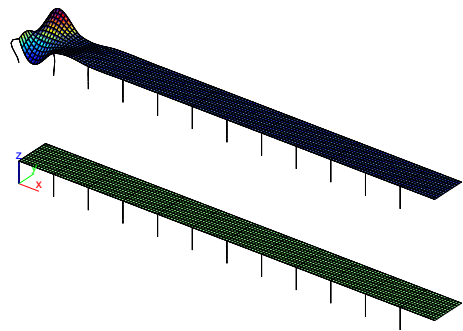

(f) $\mu= \pm 2.0317+3.1416 i$

Figure 8: The real part (upper) and imaginary part (lower) of the selected free waves of the frame at $15 \mathrm{~Hz}$.

These observations suggests that the frequency ranges with clustered modes can be predicted by analyzing the number of non-decaying free waves based on a finite element model of a single unit, avoiding the eigenvalue analysis of a full structure. This is useful in the preliminary design of an experiment. If the frequency range of interest contains bands where a large number of non-decaying waves are found, the modes appear to be closely spaced with similar mode shapes and the sensor locations should be carefully chosen in order to distinguish between them.

The aim of a multi-setup modal test is to accurately characterize the mode shapes. Usually, a few reference sensors are used for merging data in different setups and the roving sensors are typically moved from one bay or several adjacent bays to the next ones for obtaining detailed spatial modal information. If this conventional sensor placement approach is applied to identify clustered modes and the data is merged after identifying modal characteristics, two challenges arise: (1) identifying the partial modes in each setup which may be nearly identical with closely spaced natural frequencies; (2) pairing the partial modes in different setups.

\section{Optimal sensor placement strategy for multi-setup modal identification}

An optimal sensor placement strategy is proposed for multi-setup modal testing of large structures. The criterion used in this paper for the optimal sensor placement is the Information Entropy Index (IEI) [19]. A computational algorithm based on the Backward Sequential Sensor Placement (BSSP) algorithm is proposed next.

\subsection{Optimal sensor placement for single setup modal identification}

Before considering the problem of optimal sensor placement for multi-setup modal identification, the methodology of optimal sensor placement based on Information Entropy is briefly recapitulated [19]. For modal identification, the objective function is based on the uncertainty in the estimates of the modal coordinates from a set of measured responses.

Following Papadimitriou and Lombaert [19], the measured response with prediction error is written as

$$
\mathbf{x}=\mathbf{L} \Phi \boldsymbol{\theta}+\mathbf{L e}
$$


where $\boldsymbol{\Phi}$ contains mode shape vectors, $\mathbf{L}$ is the observation matrix that selects the observed response quantities, e is the prediction error vector containing modeling error and measurement error and $\boldsymbol{\theta}$ is the modal coordinate vector to be estimated. $\boldsymbol{\theta}$ can be modal displacements, modal velocities or modal accelerations depending on the type of the measured quantities.

An estimate $\boldsymbol{\theta}_{0}$ of the modal coordinates is obtained from the least-squares solution:

$$
\boldsymbol{\theta}_{0}=\left((\mathbf{L} \boldsymbol{\Phi})^{\mathrm{T}}(\mathbf{L} \boldsymbol{\Phi})\right)^{-\mathbf{1}}(\mathbf{L} \boldsymbol{\Phi})^{\mathrm{T}} \mathbf{x}
$$

The covariance matrix of the estimation error, characterizing the uncertainty on the modal coordinates, is calculated from the covariance matrix $\boldsymbol{\Sigma}_{\mathrm{t}}$ of the prediction error as

$$
\mathbf{C}=\mathbf{E}\left[\left(\boldsymbol{\theta}-\boldsymbol{\theta}_{0}\right)\left(\boldsymbol{\theta}-\boldsymbol{\theta}_{0}\right)^{\mathrm{T}}\right]=\left[(\mathbf{L} \boldsymbol{\Phi})^{\mathrm{T}}\left(\mathbf{L} \boldsymbol{\Sigma}_{\mathrm{t}} \mathbf{L}^{\mathrm{T}}\right)^{-\mathbf{1}}(\mathbf{L} \boldsymbol{\Phi})\right]^{-\mathbf{1}}
$$

The Fisher Information Matrix is the inverse of the covariance matrix:

$$
\mathbf{Q}=(\mathbf{L} \boldsymbol{\Phi})^{\mathrm{T}}\left(\mathbf{L} \boldsymbol{\Sigma}_{\mathrm{t}} \mathbf{L}^{\mathrm{T}}\right)^{-\mathbf{1}}(\mathbf{L} \boldsymbol{\Phi})
$$

The Information Entropy, as given by equation (7), is a scalar measure of the uncertainty in the estimated parameters [19]:

$$
h\left(\mathbf{L} ; \boldsymbol{\Sigma}_{\mathbf{t}}, \mathbf{D}\right)=-\int \ln p\left(\boldsymbol{\theta} \mid \boldsymbol{\Sigma}_{\mathrm{t}}, \mathbf{D}\right) p\left(\boldsymbol{\theta} \mid \boldsymbol{\Sigma}_{\mathbf{t}}, \mathbf{D}\right) d \boldsymbol{\theta}
$$

where $p\left(\boldsymbol{\theta} \mid \boldsymbol{\Sigma}_{\mathbf{t}}, \mathbf{D}\right)$ is the posterior probability density function and $\mathbf{D}$ are the measured data.

If the number of data is sufficiently large, the Information Entropy can be asymptotically approximated by $[18]$ :

$$
h\left(\mathbf{L} ; \boldsymbol{\Sigma}_{\mathrm{t}}, \mathbf{D}\right) \approx H\left(\boldsymbol{L} ; \boldsymbol{\theta}_{0}, \boldsymbol{\Sigma}_{\mathrm{t}}\right)=\frac{1}{2} N_{\boldsymbol{\theta}} \ln (2 \pi)-\frac{1}{2} \ln \left[\operatorname{det} \mathbf{Q}\left(\mathbf{L} ; \boldsymbol{\theta}_{0}, \boldsymbol{\Sigma}_{\mathrm{t}}\right)\right]
$$

where $\mathbf{Q}$ is the Fisher Information Matrix given in equation (6). In the case of the linear relationship between the response quantities $\mathbf{x}$ and the parameters $\boldsymbol{\theta}$ in equation (3), equation (8) gives an exact expression for the Information Entropy.

The Information Entropy Index (IEI) is defined as a measure of the uncertainty in $\boldsymbol{\theta}$ relative to the uncertainty obtained for a reference configuration, typically including all DOFs:

$$
\operatorname{IEI}(\mathbf{L})=\exp \left[H\left(\mathbf{L} ; \boldsymbol{\theta}_{0}, \boldsymbol{\Sigma}_{\mathrm{t}}\right)-H\left(\mathbf{L}_{\mathrm{ref}} ; \boldsymbol{\theta}_{0}, \boldsymbol{\Sigma}_{\mathrm{t}}\right)\right]=\left[\frac{\operatorname{det} \mathbf{Q}^{-1}\left(\mathbf{L} ; \boldsymbol{\theta}_{0}, \boldsymbol{\Sigma}_{\mathrm{t}}\right)}{\operatorname{det} \mathbf{Q}^{-1}\left(\mathbf{L}_{\mathrm{ref}} ; \boldsymbol{\theta}_{0}, \boldsymbol{\Sigma}_{\mathrm{t}}\right)}\right]^{1 / 2}
$$

By minimizing the IEI, the uncertainty in the estimates is minimized. Based on the Information Entropy, the BSSP and FSSP algorithms were proposed to compute the optimal sensor locations for single setup modal testing in an efficient way $[18,19]$. The BSSP algorithm starts with sensors at all possible locations and removes one sensor at a time successively by searching the sensor configuration that gives the smallest IEI from all configurations with one sensor less. The FSSP algorithm places one sensor at a time by searching the sensor configuration that gives the smallest IEI from all configurations with one sensor more.

\subsection{Optimal sensor placement strategy for multi-setup modal identification}

The problem of optimal sensor placement for multi-setup modal identification can be formulated in different ways, for example, (1) given a maximum number of available sensors and a minimum number of positions to be covered in the test, how should the reference and roving sensors be placed such that the value of the IEI is minimized, (2) given a maximum number of available sensors and a maximum value of the IEI, how should the reference and roving sensors be placed such that the number of setups is minimized. In the following, the first optimization problem is considered.

The optimization problem is solved by considering the positions of both the reference and the roving sensors simultaneously. When modal identification is carried out for each roving setup, the quality of the combined test depends on the least informative setup. The objective of the proposed strategy is to optimize 
the worst setup and thus generally make all setups good. This is formulated as a minimax problem where the maximum value of IEI over all setups is minimized. The optimization problem is formulated as:

$$
\left[\mathrm{L}_{\mathrm{ref}} ; \mathrm{L}_{\mathrm{rov}}\right]^{*}=\underset{\left[\mathrm{L}_{\mathrm{ref}}, \mathrm{L}_{\mathrm{rov}}\right]}{\arg \min } \underset{j \in\left[1, \cdots, N_{\text {setup }}\right]}{\max }\left\{\operatorname{IEI}\left(\left[\mathrm{L}_{\mathrm{ref}} ; \mathrm{L}_{\mathrm{rov}, j}\right]\right)\right\}
$$

where the objective is to minimize the maximum value of the IEI over all setups, $\mathrm{L}_{\text {ref }}$ is the configuration of the reference sensors, $\mathrm{L}_{\mathrm{rov}, j}$ is the configuration of the roving sensors in setup $j \in\left[1, \cdots, N_{\text {setup }}\right]$ with $N_{\text {setup }}$ the total number of setups, $\mathrm{L}_{\text {rov }}=\left[\mathrm{L}_{\text {rov }, 1} ; \cdots ; \mathrm{L}_{\text {rov }, N_{\text {setup }}}\right]$.

The BSSP algorithm can be adapted to solve this minimax problem. The total number of sensors available is denoted by $N_{\mathrm{t}}$, the number of reference sensors is $N_{\text {ref }}$ and the number of roving sensors is $N_{\text {rov }}=N_{\mathrm{t}}-N_{\text {ref }}$. It is noted that $N_{\text {ref }}, N_{\text {rov }}$ and $N_{\text {setup }}$ are fixed in the following algorithm.

1. Initialize: preselect $N_{\mathrm{I}}$ candidate positions $\mathrm{L}_{\mathrm{I}}$ for $N_{\text {ref }}$ reference sensors from all possible locations.

2. While number of candidate sensors $N_{\mathrm{I}}>$ number of reference sensors $N_{\text {ref }}$, do

(a) For $i=1$ to $N_{\mathrm{I}}$

i. Obtain reference sensor configuration $\mathrm{L}_{i, \mathrm{I}-1}$ by removing sensor $i$ from $\mathrm{L}_{\mathrm{I}}$

ii. For $j=1$ to $N_{\text {setup }}$

A. Select $N_{\text {rov }}$ optimal positions $\mathrm{L}_{\text {rov }, j}$ for roving sensors from all possible roving sensor positions of setup $j$, such that the sensor configuration $\left[\mathrm{L}_{i, \mathrm{I}-1} ; \mathrm{L}_{\mathrm{rov}, j}\right]$ gives the minimum IEI.

B. Obtain the IEI of configuration $\left[\mathrm{L}_{i, \mathrm{I}-1} ; \mathrm{L}_{\mathrm{rov}, j}\right]$ for setup $j: \mathrm{IEI}_{i, j}$ end

iii. Find the maximum IEI over all setups: $\max \left\{\operatorname{IEI}_{i, 1}, \cdots, \operatorname{IEI}_{i, N_{\text {setup }}}\right\}$

end

(b) Select the reference sensor configuration $\mathrm{L}_{\mathrm{opt}, \mathrm{I}-1}$ that minimizes the maximum IEI over all setups

(c) $\mathrm{L}_{\mathrm{I}}=\mathrm{L}_{\mathrm{opt}, \mathrm{I}-1}, N_{\mathrm{I}}=N_{\mathrm{I}}-1$

end

In step $1, N_{\mathrm{I}}$ candidate positions $\mathrm{L}_{\mathrm{I}}$ for reference sensors are preselected from all possible positions using the BSSP or FSSP algorithm $\left(N_{\mathrm{I}}>N_{\text {ref }}\right)$. The roving sensors are not considered in this step. From these candidate positions $\mathrm{L}_{\mathrm{I}}$, a subset of $N_{\text {ref }}$ positions for reference sensors $\mathrm{L}_{\text {ref }}$ is selected by removing one sensor at a time following step 2, where roving sensors are taken into account. In step ii, each setup consists of the reference sensors, supplemented by optimally located roving sensors, which can also be obtained using the FSSP or BSSP algorithm. This procedure will be illustrated by means of the example in subsection 4.1.

Although the IEI is used as a measure of quality of the modal testing, the proposed strategy can be adopted for other criteria depending on how the problem is addressed. If the effective independence criterion is used, for example, the algorithm for multi-setup measurement should be formulated based on the iterative algorithm in [11].

Although the strategy is initially proposed to avoid problems with data merging after modal identification, it is also applicable to other approaches where the data are merged before modal identification as it is equally important to have highly informative data in each setup.

In the following section, the optimal sensor placement strategy is applied to the repetitive frame structure with twelve bays previously considered and the office building "Blok D" of KU Leuven, which is a nearly repetitive structure. Although the focus is on repetitive structures, the proposed sensor placement strategy is general and can be applied to any structure.

\section{Applications}

\subsection{The twelve-bay repetitive frame structure}

The optimal sensor locations are studied for the repetitive frame structure with twelve bays discussed in subsection 2.1. A distinction is made between two cases of modal identification. In the first case, the modal test is performed to identify modes of the entire structure. In the second case, the aim is to have a detailed characterization of the mode shapes in one particular part of the structure. 


\subsubsection{Modal testing for identification of modes of the entire structure}

Two problems are investigated in this section: (1) the required number of reference sensors; (2) the selection of a roving sensor strategy. The roving sensors can be either located in a cluster (e.g. figure 9a) or uniformly distributed (e.g. figure $9 \mathrm{~b}$ ) over the repetitive parts of the structure.

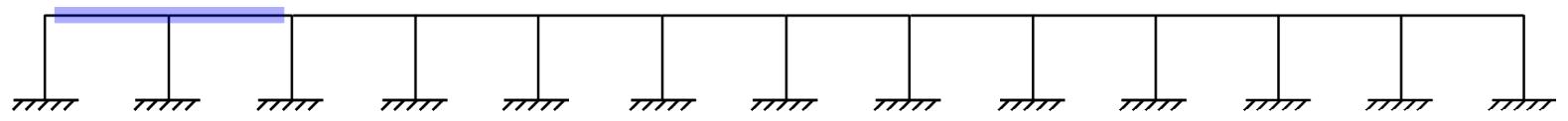

(a)

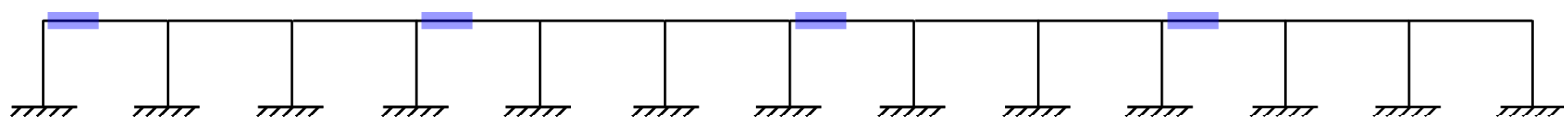

(b)

Figure 9: (a) A cluster and (b) a uniform distribution of roving sensors in one setup.

The aim of the analysis is to identify the twelve closely spaced modes in the first mode cluster (shown in figure 3). The bending of the floor is the dominant deformation pattern, thus the sensors are put in the vertical direction ( $\mathrm{z}$ direction) only. A sensor location grid, where the grid points are possible sensor locations, is predefined, as shown in figure 10.

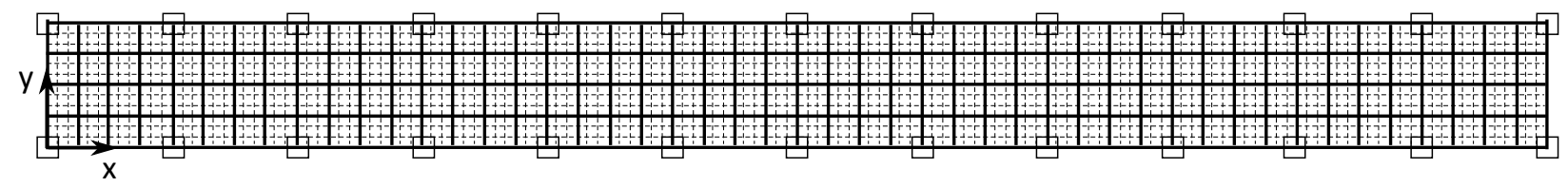

Figure 10: The sensor grid (solid lines) and the finite element mesh (dashed lines).

The roving sensor strategy illustrated in figure $9 \mathrm{a}$ is studied first. It is assumed that twenty uni-axial sensors are available $\left(N_{\mathrm{t}}=20\right)$. Three cases with a different number of setups are investigated: twelve setups, eight setups and six setups. For the case with twelve setups, the roving sensors are moved from the first to the next bay until the last bay is reached. For the case with eight setups, the roving sensors are at each time distributed over one bay and half of the adjacent bay. For the case with six setups, the roving sensors are at each time distributed over two adjacent bays. The optimal sensor locations are determined following the proposed strategy with twelve reference sensors $\left(N_{\text {ref }}=12, N_{\text {rov }}=8\right)$.

The algorithm proposed in subsection 3.2 is elaborated in detail for the case with six setups. $N_{\mathrm{I}}=30$ candidate reference sensor positions are preselected using the BSSP algorithm, shown in figure 11a, from which twelve reference sensors positions are selected as follows. First, one candidate reference sensor position is removed from the 30 positions and a configuration with 29 reference sensors $\mathrm{L}_{i, \mathrm{I}-1}$ is obtained with $i \in[1, \cdots, 30]$ referring to the candidate set of reference sensors. An example of the reference sensor configuration with one sensor less is shown in figure 11b. Second, the optimal positions of roving sensors for each setup $\mathrm{L}_{\text {rov }, j}$ are selected from all possible roving sensor positions in setup $j$ by minimizing the value of the IEI of the combined set of sensors $\left[\mathrm{L}_{i, \mathrm{I}-1} ; \mathrm{L}_{\mathrm{rov}, j}\right]$, where $j \in[1, \cdots, 6]$ indicates different setups. As an example, the optimal roving sensor positions for setup one are shown in figure 11c. The maximum value of the IEI over all setups is then picked up and taken as a measure of the optimality of the corresponding reference sensor configuration with 29 sensors. This process is repeated for all cases where a different candidate reference sensor position is removed from the 30 preselected positions, resulting in 30 reference sensor configurations. The one which minimizes the maximum value of the IEI over all setups is selected and used in the next iteration. One candidate reference sensor position is removed in each iteration until the number of reference sensors reaches 12 .

Figure 12 shows the optimal sensor locations with twelve reference sensors and clustered roving sensors. It can be seen that the optimized reference sensor locations are uniformly distributed over the structure. 


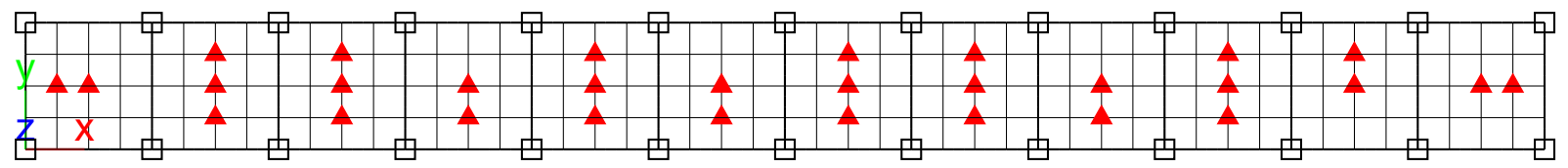

(a)

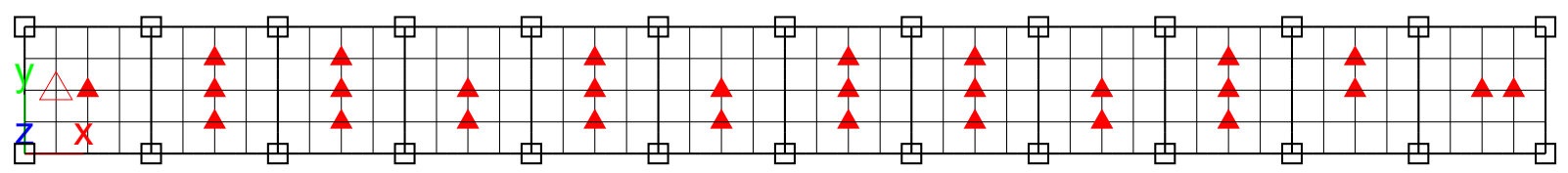

(b)

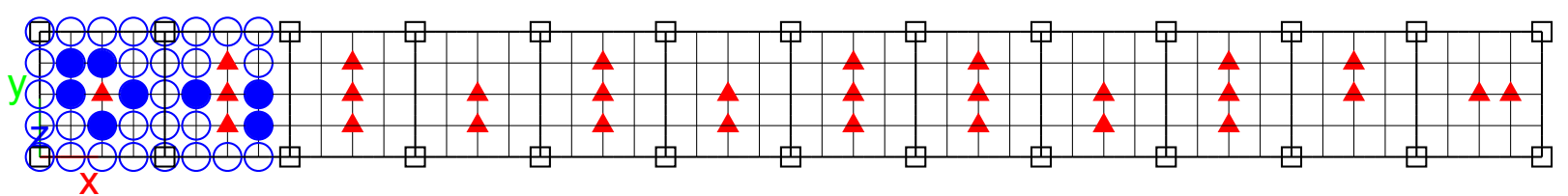

(c)

Figure 11: (a) Preselected candidate positions for reference sensors $\mathrm{L}_{\mathrm{I}}$; (b) An example of the reference sensor configuration $\mathrm{L}_{i, \mathrm{I}-1}$ with one sensor less (solid triangles), $i \in[1, \cdots, 30]$; the removed sensor (hollow triangle); (c) The 8 optimally located roving sensors $\mathrm{L}_{\mathrm{rov}, j}$ (solid circles) in addition to the reference sensor configuration $\mathrm{L}_{i, \mathrm{I}-1}$ (solid triangles), selected from all possible roving sensor locations (hollow circles) in setup $j=1$.

The second roving sensor strategy (figure $9 \mathrm{~b}$ ) is evaluated using the same twelve-bay structure with modal tests performed in twelve setups, eight setups and six setups. The roving sensors are placed on four separated areas in one setup and moved to the next four separated areas until they cover the entire structure. Figure 13 shows the optimal sensor locations with twelve reference sensors and uniformly distributed roving sensors.

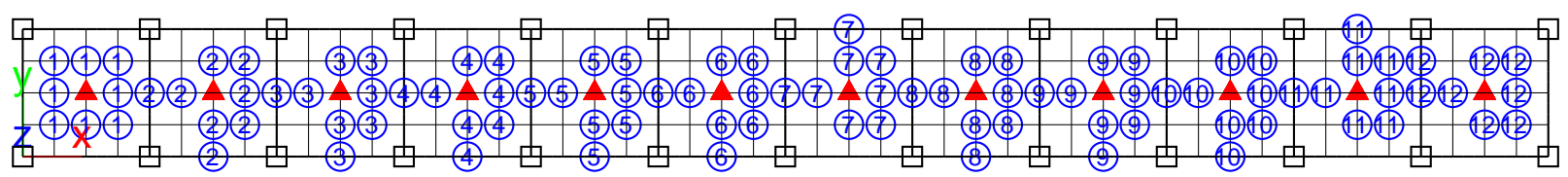

(a)

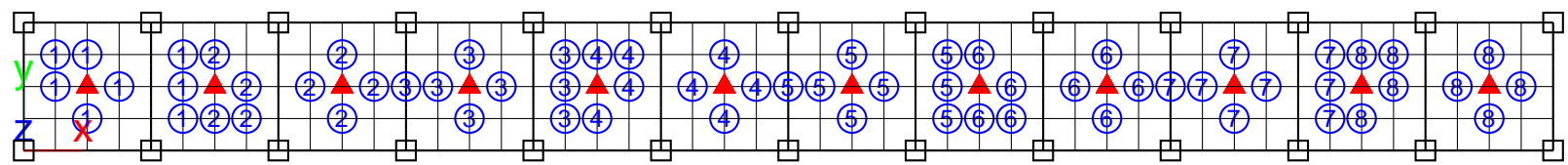

(b)

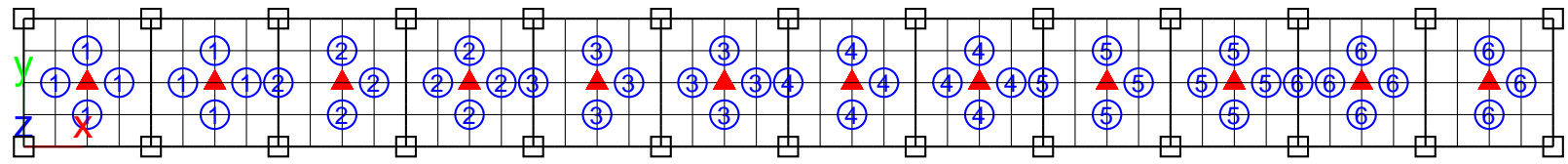

(c)

Figure 12: Optimal locations of the twelve reference sensors (triangles) and the clustered roving sensors (circles) for a modal testing in (a) twelve setups, (b) eight setups and (c) six setups. 


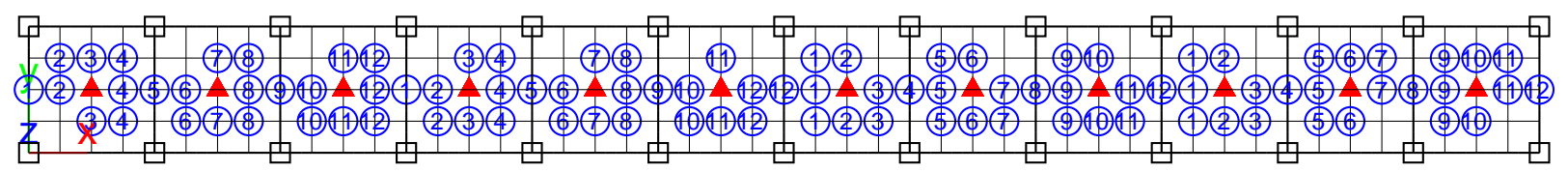

(a)

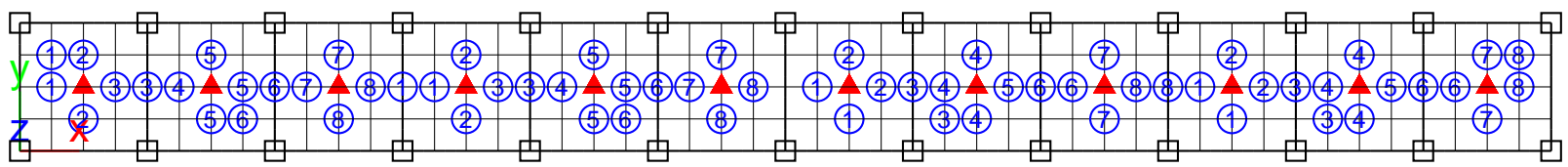

(b)

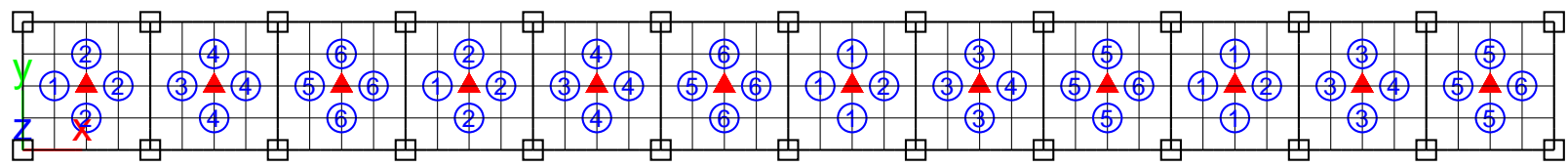

(c)

Figure 13: Optimal locations of the twelve reference sensors (triangles) and the uniformly distributed roving sensors (circles) for a modal testing in (a) twelve setups, (b) eight setups and (c) six setups.

The influence of the number of reference sensors on the value of the IEI is now investigated. In practice, there are constraints on the number of sensor available for the modal test and the measurement time while a sufficient number of positions needs to be covered to accurately characterize the mode shapes. The total number of sensors $N_{\mathrm{t}}$, the number of covered positions $N_{\text {cover }}$, the number of reference sensors $N_{\text {ref }}$ and the number of setups $N_{\text {setup }}$ are related as follows:

$$
N_{\text {cover }}=N_{\text {ref }}+N_{\text {setup }}\left(N_{\mathrm{t}}-N_{\text {ref }}\right)
$$

where $N_{\mathrm{t}}-N_{\text {ref }}$ is the number of roving sensors. Table 1 shows the number of positions covered as a function of the number of setups and the number of reference sensors when twenty sensors are available $\left(N_{\mathrm{t}}=20\right)$.

\begin{tabular}{ccccccccccc}
\hline$N_{\text {ref }}$ & 7 & 8 & 9 & 10 & 11 & 12 & 13 & 14 & 15 & 16 \\
\hline$N_{\text {cover }}$ (12 setups) & 163 & 152 & 141 & 130 & 119 & 108 & 97 & 86 & 75 & 64 \\
$N_{\text {cover }}$ (8 setups) & 111 & 104 & 97 & 90 & 83 & 76 & 69 & 62 & 55 & 48 \\
$N_{\text {cover }}$ (6 setups) & 85 & 80 & 75 & 70 & 65 & 60 & 55 & 50 & 45 & 40 \\
\hline
\end{tabular}

Table 1: The number of positions covered when twenty sensors are available.

The number of reference sensors is now varied from seven to sixteen. The optimal locations of the reference and roving sensors are determined using the proposed optimal sensor placement strategy. The value of the IEI is plotted as a function of the number of reference sensors in figure 14. It is found that the value of the IEI decreases with the number of reference sensors. For the roving sensor strategy with clustered roving sensors, it can be seen that the value of the IEI decreases rapidly when the number of reference sensors increases from seven to twelve. A low value of the IEI indicates a small uncertainty in the estimated modal coordinates. The number of reference sensors is preferably equal to or larger than twelve, which is the number of modes. It is also observed that the value of the IEI is generally larger when the number of setups is larger. This is because each setup covers a smaller floor area when the number of setups is larger and it is therefore more difficult to distinguish between the modes. Therefore the number of reference sensors should be maximized and the number of setups should be minimized in a multi-setup modal test. When the number of reference sensors is less than twelve, the uniformly distributed roving sensors generally lead to a much smaller value of the IEI than in case of the clustered roving sensors. The measurement quality is therefore largely improved. When the number of reference sensors is equal to or larger than twelve, the two roving sensor strategies provide similar results in the same order of magnitude. 


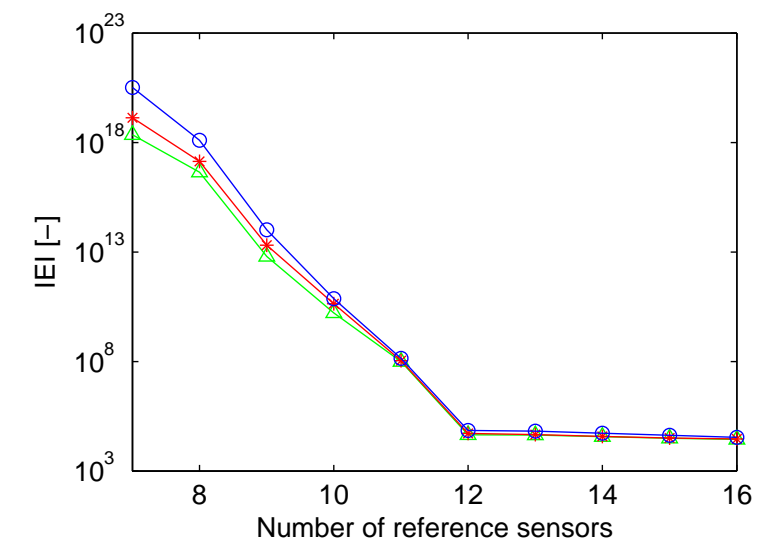

(a)

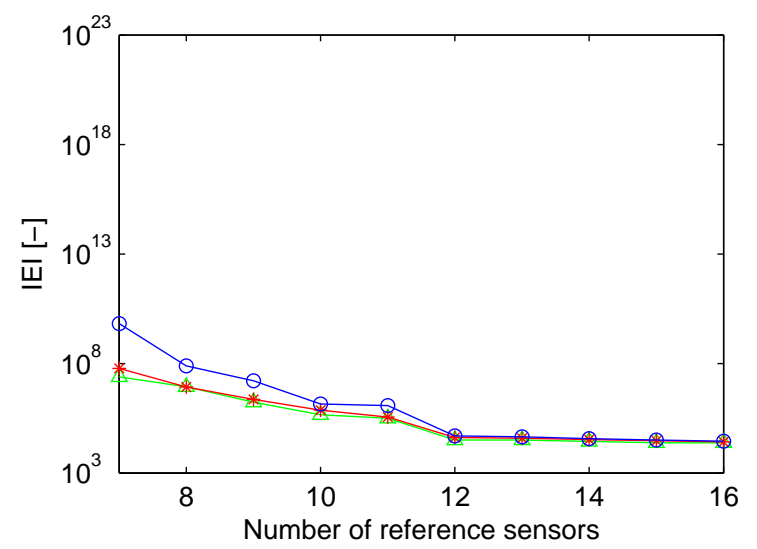

(b)

Figure 14: The value of the IEI as a function of the number of reference sensors for the roving sensor strategy with (a) clustered roving sensors and (b) uniformly distributed roving sensors in twelve setups (circles), eight setups (asterisks) and six setups (triangles).

Theoretically, if the number of observed DOFs is less than the number of modes to be identified, the observed mode shape vectors are linearly dependent. In this case, the term $(\mathbf{L} \boldsymbol{\Phi})^{\mathrm{T}}(\mathbf{L} \boldsymbol{\Phi})$ in equation 4 is not invertible. This makes it impossible to distinguish between the modes and also implies that important information required for identifying these modes is missing. For multi-setup modal testing, the total number of sensors in one setup, including both reference and roving sensors, should at least be equal to the number of modes to enable the distinction of the modes in this setup. This, however, does not guarantee that the modes can be distinguished in this setup. Since the roving sensors are constrained to particular positions for each setup, they may not contribute to distinguishing between the observed modes. The number of reference sensors is therefore preferably at least equal to the number of modes.

A uniform distribution of roving sensors is generally superior over a cluster of roving sensors, since some modes may appear nearly identical in some adjacent units of a repetitive structure.

The best sensor placement plan is now determined for the twelve-bay frame structure to identify the twelve modes in the first mode cluster (between $14.0 \mathrm{~Hz}$ and $15.3 \mathrm{~Hz}$ ). The required number of positions covered is determined by the smallest wavelength in the frequency range of interest. The smaller the wavelength is, the more positions need to be covered to obtain accurate mode shapes. Since the dominant deformation pattern of the mode shapes in the frequency range of interest is vertical bending, the vertical bending non-decaying free wave is the main component of the mode shapes. The smallest wavelength in the first mode cluster is similar to the vertical bending non-decaying free wave at $15 \mathrm{~Hz}$ (figure 8c), which is around $10 \mathrm{~m}$ (two spans). If fourteen sensors per wavelength are needed to accurately represent the mode shapes, the total number of covered positions should be $N_{\text {cover }}=14 \times 6=84$ for the twelve-bay frame. From all cases considered in table 1, those with more than 84 covered positions should be considered. In case the modal testing is performed in twelve setups, the number of reference sensors can be taken as twelve or more as long as the number of covered positions is larger than 84. If the number of setups is limited to eight due to limits on the measurement time, there are two options: ten reference sensors and eight setups or seven reference sensors and six setups. The latter, however, gives worse results. Therefore the case with ten reference sensors and eight setups should be taken. In this case, a uniform distribution of roving sensors is preferable over a cluster of roving sensors.

It is concluded that the number of reference sensors is preferably equal to or larger than the number of modes to be identified and the number of setups depends on the required number of positions covered. If this is not possible, it is preferable to use as many reference sensors as possible. In this case, the roving sensor strategy has a large influence on the IEI and a uniform distribution of roving sensors is preferred. The influence of the roving sensor strategy is very small when the number of reference sensors is equal to or larger than the number of modes. 


\subsubsection{Modal testing for identification of modes of a particular part of the structure}

In some cases, the aim of the modal identification is to obtain detailed mode shapes of a particular part of a structure, e.g. one particular floor in a large building. When sensors are only positioned on that particular part, it is difficult to distinguish between modes which appear to be similar there. It is therefore important to have a sufficiently large number of reference sensors distributed over the entire structure. The roving sensors are positioned on the particular part of interest to obtain detailed mode shapes.

The optimal sensor locations are determined for the twelve modes (figure 3) in the first mode cluster (between $14.0 \mathrm{~Hz}$ and $15.3 \mathrm{~Hz}$ ) of the two central bays of the twelve-bay frame structure (figure 15). Twenty uni-axial sensors are available, roving sensors are moved in three setups located at the two central bays. The optimal locations of the three setups including the twelve reference sensors are shown in figure 16. The reference sensors are distributed over the entire structure. The value of the IEI is plotted as a function of the number of reference sensors in figure 17. The value of the IEI decreases with the number of reference sensors. The number of reference sensors is preferably equal to or larger than twelve in order to make the modes distinguishable, as stated in subsection 4.1.1.

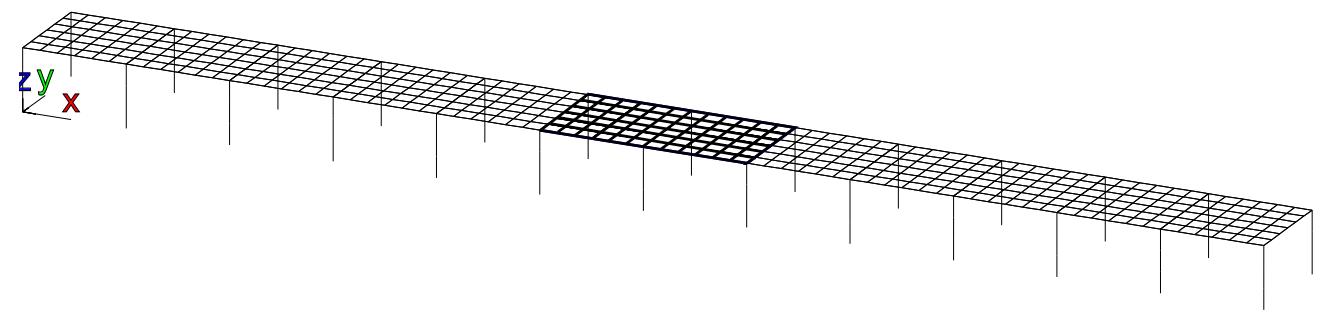

Figure 15: The two central bays of the twelve-bay structure.

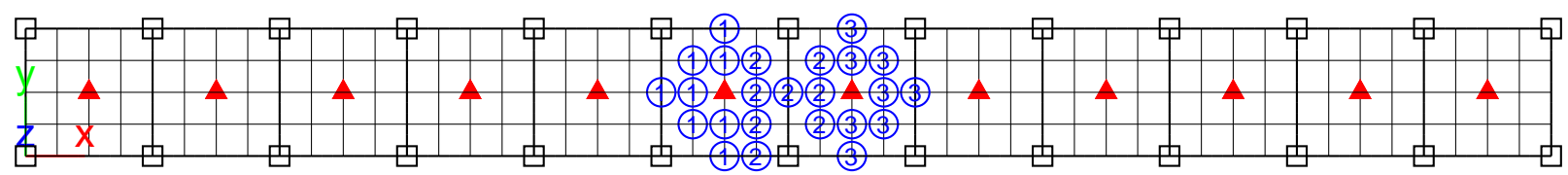

Figure 16: Optimal locations of the twelve reference sensors (triangles) and roving sensors (circles) for identifying the modes of the two central bays.

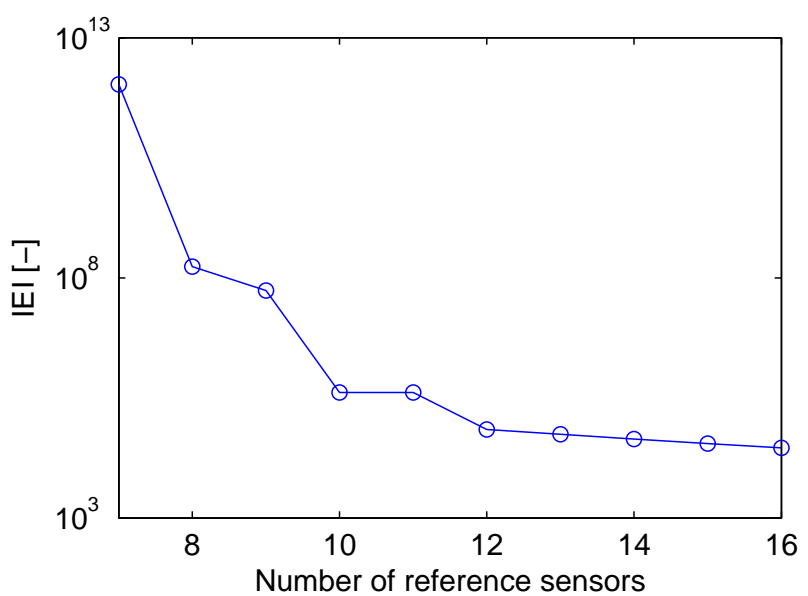

Figure 17: The value of the IEI as a function of the number of reference sensors when the sensor locations are optimized for the identification of the modes of the two central bays. 


\subsection{The office building "Blok D" of KU Leuven}

An experimental modal test was performed on the office building "blok D" of KU Leuven located at Willem de Croylaan 54, 3001 Leuven, Belgium. The proposed optimal sensor placement strategy for multisetup modal analysis is validated by the results from this experiment.

\subsubsection{Measurement on the "Blok D" building of $K U$ Leuven}

Figure 18 shows a picture of the "Blok D" building, which is a three-story building (floors 0 - 2) with basement, connected to a neighboring building through a hallway and a corridor on the ground floor 0 . The load-bearing structure is composed of reinforced concrete beams, columns and floor slabs. A right-handed coordinate system is used with $\mathrm{x}$-axis in the direction of the short side of the floor plan, $\mathrm{y}$-axis in the direction of the long side and z-axis perpendicular to the floor plan, as shown in figures 18 and 19. The building has a nearly repetitive structural layout in the y-direction.

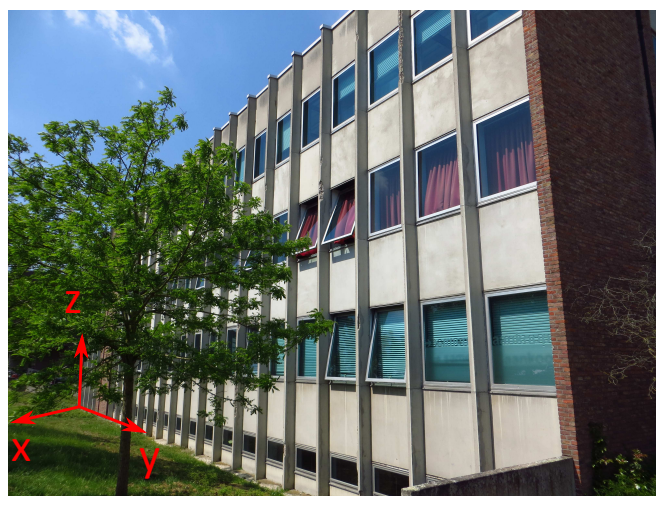

Figure 18: The "Blok D" building of KU Leuven.

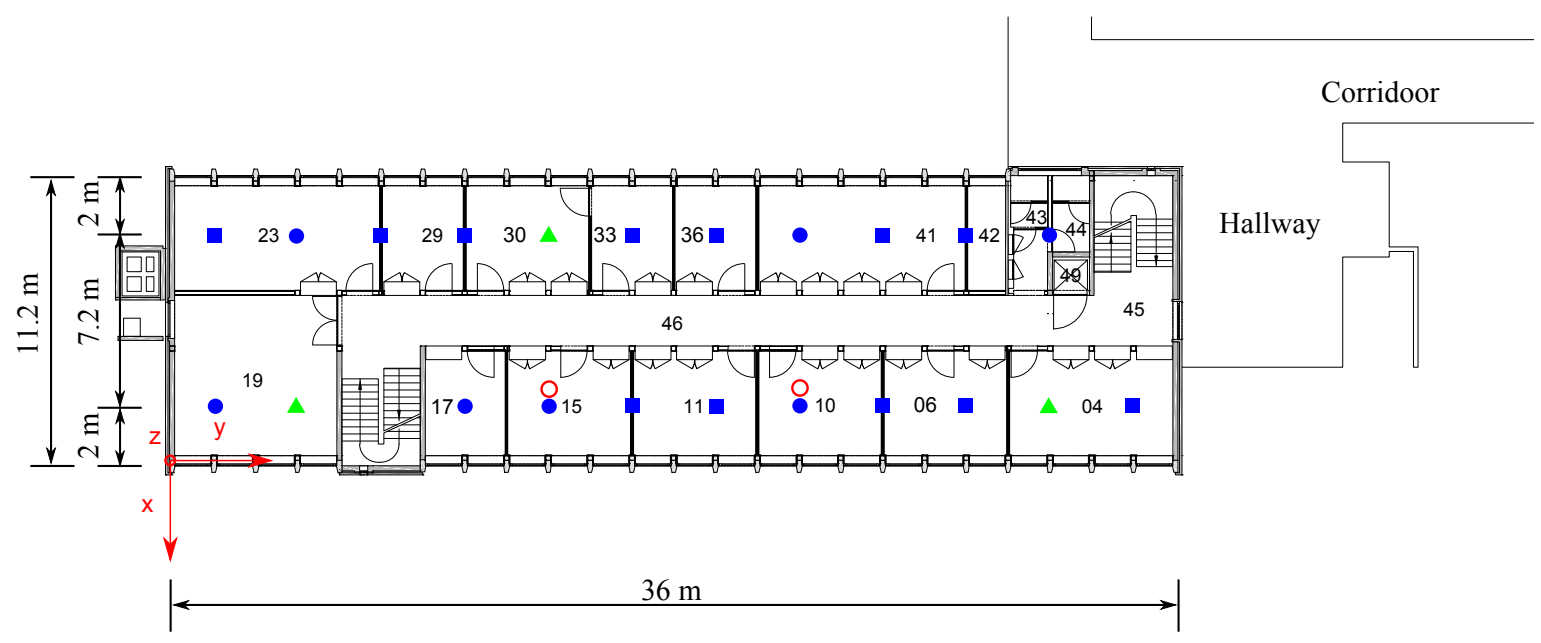

Figure 19: The plan of floor 2 and the measurement setup with 12 GeoSIG GMS-18 units (solid squares), 3 GeoSIG GMS-plus units (solid triangles), 7 uni-axial accelerometers (solid circles) and 2 hammer locations (hollow circles).

The measurements were performed on the top floor 2. The locations of the excitations and the measured responses on floor 2 are shown in figure 19. Repeated hammer excitation was simultaneously applied in the vertical direction at two locations on the floor and the acceleration responses were measured at 22 locations. 
Fifteen wireless tri-axial acceleration sensors including twelve GeoSIG GMS-18 units and three GeoSIG GMSplus units were used. The synchronization of the GeoSIG units is provided through GPS. Each triaxial sensor has three channels measuring accelerations in $\mathrm{x}-, \mathrm{y}$ - and z-direction. In addition, each GeoSIG GMSplus has three additional channels. Seven uni-axial accelerometers in the z-direction and the two hammers were connected to the additional channels of the GeoSIG GMSplus units. Fifty-two channels were measured in total, including two hammer forces, fifteen accelerations in the x-direction, fifteen accelerations in the $\mathrm{y}$-direction and twenty-two accelerations in the z-direction,.

Modal identification was performed using MACEC [30], a Matlab toolbox for modal analysis of structures. The vertical bending deformation of floor 2 is of our interest, therefore only the twenty-two accelerations in the z-direction are taken into account in the modal identification. Since the accelerations in the zdirection at the excitation locations exceeded the gravity acceleration $g$ and the corresponding senors were disconnected with the floor, these two accelerations were not considered. Therefore, the modal identification was performed with twenty acceleration channels and two force channels.

The data-driven reference-based combined deterministic-stochastic subspace identification (CSI/ref) method was applied [31]. A value of 50 was selected for the half of the number of block rows $i$ of the Hankel matrices and the maximum model order is 120 . The stabilization criteria were selected as follows: the differences in natural frequency, damping ratio, mode shape and modal transfer norm were set as 1\%, $5 \%, 1 \%$ and $50 \%$, respectively; the damping range and the number of highest modal transfer norms were set as $0-50 \%$ and 60, respectively; the Modal Phase Collinearity (MPC) lower bound, the Mean Phase (MP) and Mean Phase Deviation (MPD) upper bounds were set to $0.5,90^{\circ}$ and $90^{\circ}$, respectively.

The stabilization diagram considering all twenty response locations is shown in figure 20, showing how 8 modes could be identified. The natural frequencies, modal damping ratios and Modal Phase Collinearity (MPC) values are listed in table 2. The MPC values of mode 5 and mode 7 are relatively low, indicating that these two modes are not well identified. The mode shapes are plotted in figure 21, where mode 1 and mode 3 have similar mode shapes ( $1^{\text {st }}$ bending mode) and the same is observed for mode 2 and mode 4 ( $2^{\text {nd }}$ bending mode).

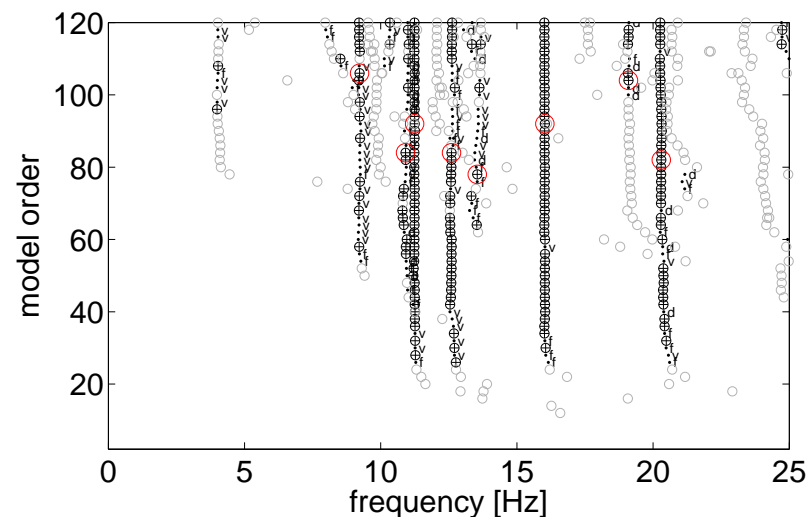

Figure 20: The stabilization diagram with picked modes (large hollow circles). 


\begin{tabular}{cccc}
\hline Mode & $f[\mathrm{~Hz}]$ & $\xi[\%]$ & MPC \\
\hline 1 & 9.22 & 2.2 & 0.85 \\
2 & 10.91 & 3.8 & 0.94 \\
3 & 11.25 & 3.0 & 0.94 \\
4 & 12.60 & 3.3 & 0.96 \\
5 & 13.54 & 2.2 & 0.71 \\
6 & 16.03 & 2.5 & 0.95 \\
7 & 19.10 & 3.4 & 0.67 \\
8 & 20.30 & 2.9 & 0.85 \\
\hline
\end{tabular}

Table 2: The natural frequencies, modal damping ratios and MPC values of the identified modes.
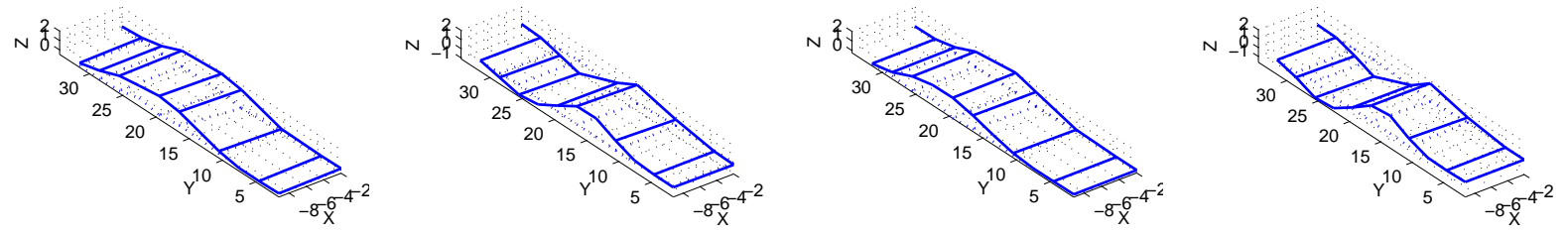

(a) mode 1

(b) mode 2

(c) mode 3

(d) mode 4
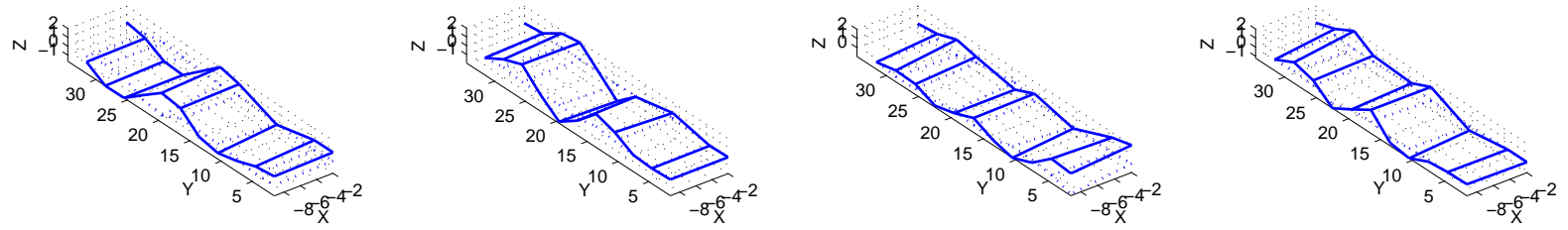

(e) mode 5

(f) mode 6

(g) mode 7

(h) mode 8

Figure 21: The eight modes identified from all twenty acceleration channels.

\subsubsection{Experimental validation of the proposed optimal sensor placement strategy}

Four multi-setup modal tests are mimicked from the original experiment in order to validate the optimal sensor placement strategy for multi-setup modal analysis. It is assumed that a total of five acceleration sensors are available and two of them are used as reference sensors. The other three sensors are roving sensors which are moved from one setup to the next until they cover all the twenty measured locations of the original test, resulting in six setups in one assumed multi-setup modal test.

The proposed optimal sensor placement strategy is applied to select the optimal reference and roving sensor locations from the twenty locations of the original test. The mode shapes are needed in the calculation of the Information Entropy Index, which is the optimal sensor placement criterion used in the proposed strategy. Usually, a preliminary finite element model is built to calculate the modes. In this study, however, the eight modes identified from the original test, as illustrated in figure 21, are used in the calculation of the Information Entropy Index and the optimal sensor locations. Figure 22b shows the optimally selected reference sensor locations and the roving sensors that are moved in clusters from setup 1 to setup 6 (case OC). The case with uniformly distributed roving sensors is also considered. While the reference sensors are kept at the same optimal locations as in figure $22 \mathrm{~b}$, the roving sensors are uniformly distributed over the structure, shown in figure $22 \mathrm{~d}$ (case OU). As a contrast, badly chosen reference sensor locations are determined by maximizing the maximum value of the IEI over all setups, shown in figure 22a for clustered roving sensors (case BC) and figure 22c for uniformly distributed roving sensors (case BU). 


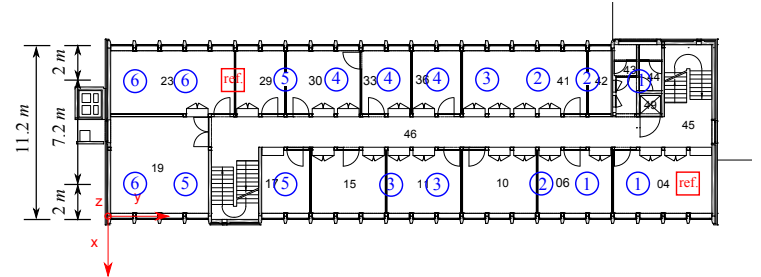

(a) case BC: badly chosen reference sensor locations and clustered roving sensors

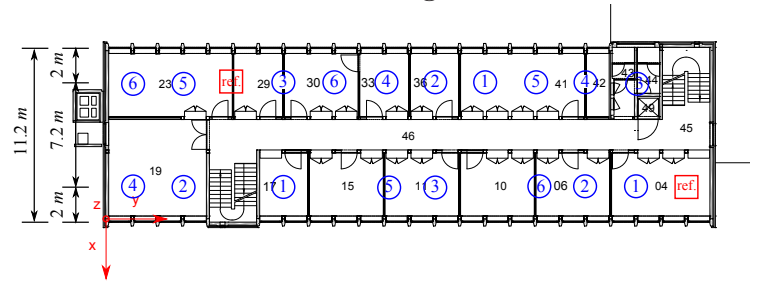

(c) case BU: badly chosen reference sensor

locations and uniformly distributed roving sensors

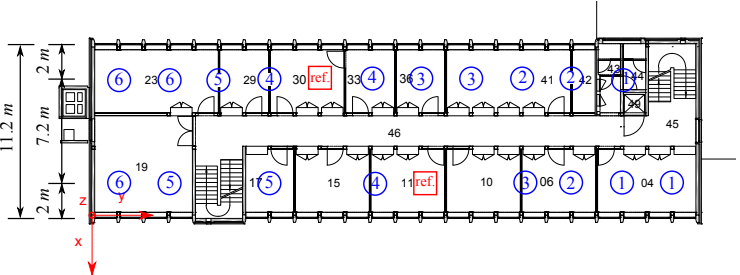

(b) case OC: optimally selected reference sensor locations and clustered roving sensors

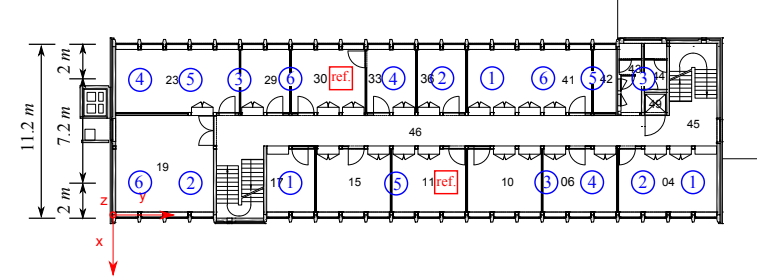

(d) case OU: optimally selected reference sensor locations and uniformly distributed roving sensors

Figure 22: The reference (squares) and roving (circles) sensor locations of the 4 assumed measurement cases.

Modal identification is carried out for all the four assumed cases. The PoSER data merging approach [3] is applied, where modal identification is performed for each setup using the same procedure in subsection 4.2.1 and the identified partial modes are merged to obtain the global modes. Figure 23 shows the stabilization diagrams of setup 2 and setup 6 for the four assumed cases. For the case with badly chosen reference sensors locations and clustered roving sensors, the stabilization diagram in setup 6 is not clear and only two stable modes can be picked up. All eight modes seem to appear in setup 6 for cases OC, BU and OU, while the stability of each mode differs from case to case. For setup 2, mode 2 does not appear for cases BC and BU and mode 7 does not appear clearly for cases BC and OC.

The natural frequencies and modal damping ratios of the identified modes are listed in table 3 . The MAC values between the modes identified from the assumed cases and the corresponding modes identified from the original measurements considering all twenty measured locations are calculated, shown in table 4. The MPC values are also shown in table 4 indicating the complexity of the modes. Only the modes which could be identified in all setups are considered here. For case BC, only modes 4, 6 and 8 are identified in all setups. The MAC value between mode 4 identified here and mode 4 from the original measurements is very low (0.57), indicating that this mode is not well identified. For case OC, modes 1, 3, 4, 6 and 8 are well identified with relatively high MPC and MAC values. Modes 2 and 5 are also identified with slightly lower MPC and MAC values. For case BU, modes 6 and 8 are well identified. Modes 1, 3, 4 and 5 are identified with less accuracy. For case OU, all modes except for mode 7 are well identified.

By comparing all cases, it is found that case OU gives the best results, case OC is the second best and case BC is the worst. These experiments confirm that a well selected reference sensor configuration is very important in the multi-setup modal identification. The results show that the sensor locations found with the optimal sensor placement strategy for multi-setup modal testing allow extracting more information from the measurement data. It is also found that it is better to uniformly distribute the roving sensors rather than clustering them. 

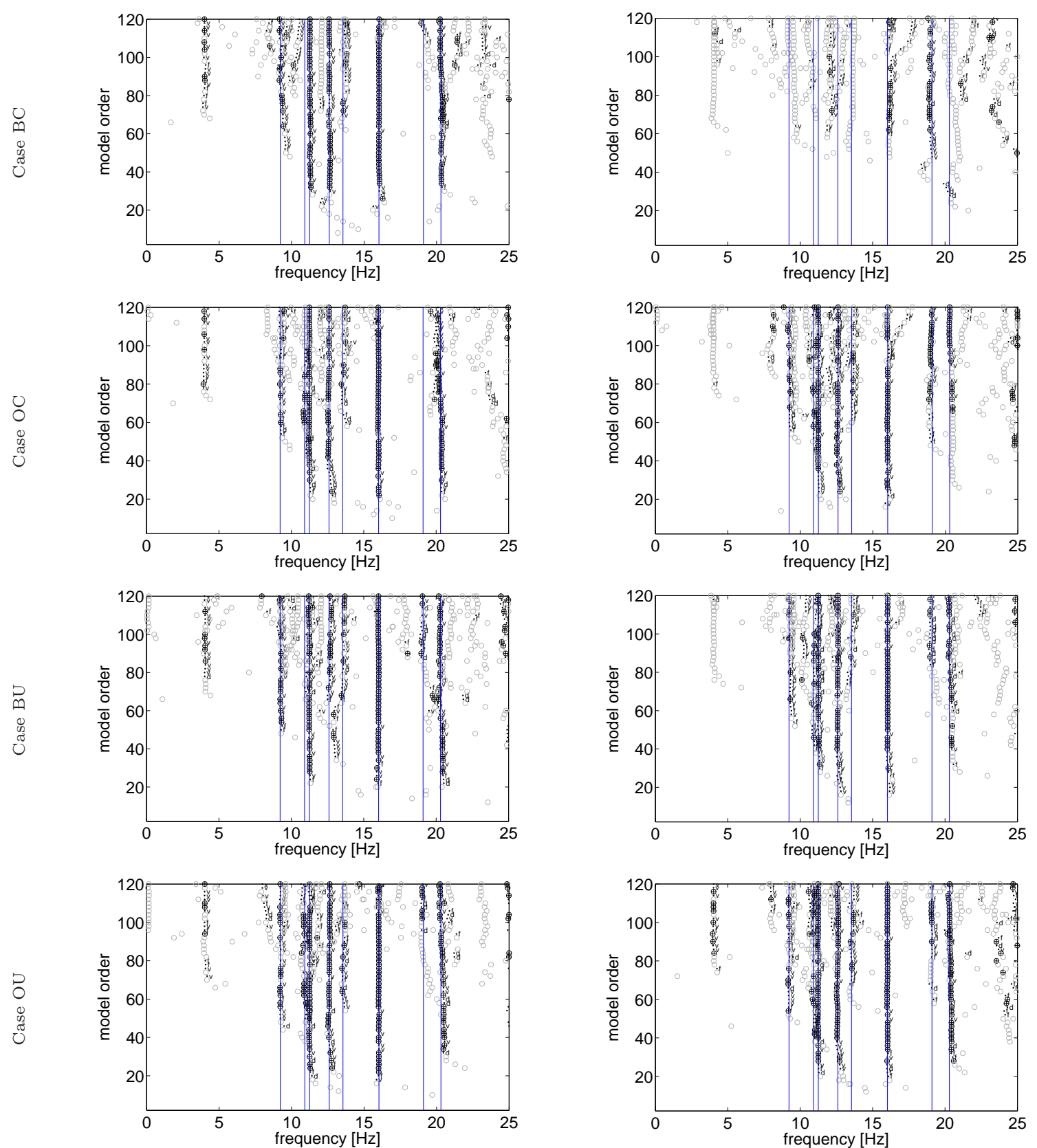

(a) setup 2

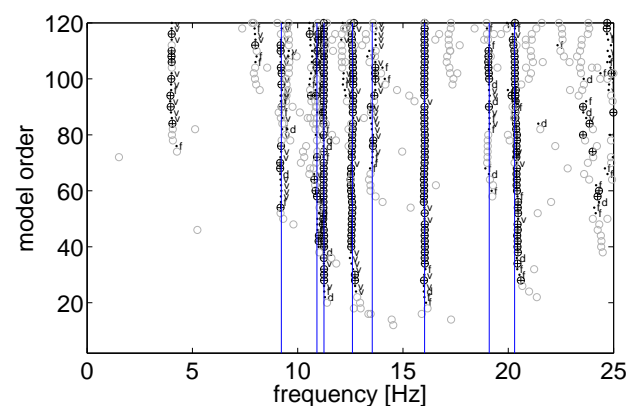

(b) $\operatorname{setup} 6$

Figure 23: The stabilization diagrams of all four assumed cases for (a) setup 2 and (b) setup 6 . The vertical lines indicate the natural frequencies identified from the original measurements. 


\begin{tabular}{|c|c|c|c|c|c|c|c|c|c|c|}
\hline Mode & $f[\mathrm{~Hz}]$ & $f_{\mathrm{bc}}[\mathrm{Hz}]$ & $f_{\mathrm{oc}}[\mathrm{Hz}]$ & $f_{\mathrm{bu}}[\mathrm{Hz}]$ & $f_{\text {ou }}[\mathrm{Hz}]$ & $\xi \xi[\%]$ & $\xi_{\mathrm{bc}}[\%]$ & $\xi_{\text {oc }}[\%]$ & $\xi_{\mathrm{bu}}[\%]$ & $\xi_{\text {ou }}[\%]$ \\
\hline 1 & 9.22 & - & 9.26 & 9.26 & 9.24 & 2.2 & - & 2.5 & 2.3 & 1.4 \\
\hline 2 & 10.91 & - & 10.92 & - & 10.90 & 3.8 & - & 4.4 & - & 3.6 \\
\hline 3 & 11.25 & - & 11.25 & 11.24 & 11.23 & 3.0 & - & 2.9 & 2.9 & 2.9 \\
\hline 4 & 12.60 & 12.54 & 12.60 & 12.61 & 12.60 & 3.3 & 3.3 & 3.3 & 3.4 & 3.4 \\
\hline 5 & 13.54 & - & 13.58 & 13.61 & 13.60 & 2.2 & - & 2.5 & 1.7 & 2.4 \\
\hline 6 & 16.03 & 16.05 & 16.01 & 16.02 & 16.01 & 2.5 & 2.5 & 2.3 & 2.4 & 2.4 \\
\hline 7 & 19.10 & - & - & - & - & 3.4 & - & - & - & - \\
\hline 8 & 20.30 & 20.38 & 20.26 & 20.27 & 20.24 & 2.9 & 2.4 & 2.7 & 2.5 & 2.5 \\
\hline
\end{tabular}

Table 3: The natural frequencies and damping ratios identified from the original measurements $(f, \xi)$, case BC $\left(f_{\mathrm{bc}}, \xi_{\mathrm{bc}}\right)$, case $\mathrm{OC}\left(f_{\mathrm{oc}}, \xi_{\mathrm{oc}}\right)$, case BU $\left(f_{\mathrm{bu}}, \xi_{\mathrm{bu}}\right)$ and case OU $\left(f_{\text {ou }}, \xi_{\text {ou }}\right)$.

\begin{tabular}{|c|c|c|c|c|c|c|c|c|c|c|}
\hline Mode & $\mathrm{MPC}$ & $\mathrm{MPC}_{\mathrm{bc}}$ & $\mathrm{MPC}_{\mathrm{oc}}$ & $\mathrm{MPC}_{\mathrm{bu}}$ & $\mathrm{MPC}_{\mathrm{ou}}$ & $\mathrm{MAC}$ & $\mathrm{MAC}_{\mathrm{bc}}$ & $\mathrm{MAC}_{\mathrm{oc}}$ & $\mathrm{MAC}_{\mathrm{bu}}$ & $\mathrm{MAC}_{\mathrm{ou}}$ \\
\hline 1 & 0.85 & - & 0.86 & 0.64 & 0.89 & 1.00 & - & 0.96 & 0.90 & 0.97 \\
\hline 2 & 0.94 & - & 0.72 & - & 0.75 & 1.00 & - & 0.87 & - & 0.96 \\
\hline 3 & 0.94 & - & 0.94 & 0.73 & 0.96 & 1.00 & - & 0.98 & 0.89 & 0.99 \\
\hline 4 & 0.96 & 0.78 & 0.96 & 0.65 & 0.97 & 1.00 & 0.57 & 0.99 & 0.82 & 0.99 \\
\hline 5 & 0.71 & - & 0.73 & 0.17 & 0.77 & 1.00 & - & 0.81 & 0.83 & 0.90 \\
\hline 6 & 0.95 & 0.94 & 0.96 & 0.90 & 0.95 & 1.00 & 0.97 & 1.00 & 0.96 & 1.00 \\
\hline 7 & 0.67 & - & - & - & - & 1.00 & - & - & - & - \\
\hline 8 & 0.85 & 0.91 & 0.86 & 0.61 & 0.85 & 1.00 & 0.91 & 0.98 & 0.91 & 0.98 \\
\hline
\end{tabular}

Table 4: The MPC values and MAC values for the identified modes from the original measurements (MPC, MAC), case BC $\left(\mathrm{MPC}_{\mathrm{bc}}, \mathrm{MAC}_{\mathrm{bc}}\right)$, case $\mathrm{OC}\left(\mathrm{MPC}_{\mathrm{oc}}, \mathrm{MAC}_{\mathrm{oc}}\right)$, case $\mathrm{BU}\left(\mathrm{MPC}_{\mathrm{bu}}, \mathrm{MAC}_{\mathrm{bu}}\right)$ and case $\mathrm{OU}\left(\mathrm{MPC}_{\mathrm{ou}}, \mathrm{MAC}_{\mathrm{ou}}\right)$.

\section{Conclusions}

This paper presents an optimal sensor placement strategy for multi-setup modal testing, which optimizes the locations of reference and roving sensors simultaneously. The procedure is illustrated for selecting optimal positions of uni-axial sensors for a repetitive frame structure with clustered modes. It is found that the number of reference sensors is preferably equal to or larger than the number of modes to be identified and the number of setups depends on the required number of positions covered. In this case, the information content, as quantified by the Information Entropy, is not very sensitive to the roving sensor strategy. When this is not possible, it is preferable to use as many reference sensors as possible and to distribute the roving sensors uniformly over the structure rather than clustering them. Clustering the roving sensors is more likely to lead to problems with distinguishing between modes with closely spaced natural frequencies which may exhibit similar modal displacements in adjacent units of the structure. The proposed strategy has been validated by an experimental modal test on the office building "Blok D" of KU Leuven, which has a nearly repetitive structural layout. It is found that the sensor locations determined by the proposed strategy allow extracting more information from the data. In this case, it is better to uniformly distribute the roving sensors rather than clustering them. This conclusion is likely to also hold for other repetitive structures where clustered modes appear. For non-repetitive structures, it is much more difficult to predict on beforehand what roving strategy will be best suited and the proposed optimization framework can be applied to select the best positions for reference and roving sensors.

\section{Acknowledgments}

The research presented in this paper has been performed within the framework of the project OT/13/59 "Quantifying and reducing uncertainty in structural dynamics", funded by the Research Council of KU Leuven. The financial support of KU Leuven is gratefully acknowledged. The second, third, fourth and last 
author are members of the KU Leuven-BOF PFV/10/002 OPTEC-Optimization in Engineering Center. The authors would also like to thank Manthos Papadopoulos and Avisek Mukherjee for the collaboration in the experiments.

\section{References}

[1] W. Heylen, S. Lammens, P. Sas, Modal analysis theory and testing, Department of Mechanical Engineering, Katholieke Universiteit Leuven, Leuven, Belgium, 1997.

[2] D.J. Ewins, Modal testing, second edition, Research Studies Press, Baldock, U.K., 2000.

[3] E. Reynders, F. Magalhães, G. De Roeck, Á. Cunha, Merging strategies for multi-setup operational modal analysis: application to the Luiz I steel arch bridge, in: Proceedings of IMAC 27, the International Modal Analysis Conference, Orlando, FL, 2009.

[4] M. Arnst, D. Clouteau, H. Chebli, R. Othman, G. Degrande, A non-parametric probabilistic model for ground-borne vibrations in buildings, Probabilistic Engineering Mechanics 21 (1) (2006) 18-34.

[5] L. Brillouin, Wave propagation in periodic structures: electric filters and crystal lattices, MacGraw-Hill, New York, 1946.

[6] D.J. Mead, Wave propagation in continuous periodic structures: research contributions from Southampton, 1964 - 1995 , Journal of Sound and vibration 190 (3) (1996) 495-524

[7] D.J. Mead, A general theory of harmonic wave propagation in linear periodic systems with multiple coupling, Journal of Sound and vibration 27 (2) (1973) 235-260

[8] G. Sen Gupta, Natural flexural waves and the normal modes of periodically-supported beams and plates, Journal of Sound and vibration 13 (1) (1970) 89-101.

[9] D.J. Mead, Wave propagation and natural modes in periodic systems: II. Multi-coupled systems, with and without damping, Journal of Sound and vibration 40 (1) (1975) 19-39.

[10] P.C. Shah, F.E. Udwadia, A methodology for optimal sensor locations for identification of dynamic systems, Journal of Applied Mechanics 45 (1) (1978) 188-196.

[11] D.C. Kammer, Sensor placement for on-orbit modal identification and correlation of large space structures, Journal of Guidance, Control, and Dynamics 14 (2) (1991) 251-259.

[12] M. Papadopoulos, E. Garcia, Sensor placement methodologies for dynamic testing, AIAA Journal 36 (2) (1998) $256-263$.

[13] F.E. Udwadia, Methodology for optimum sensor locations for parameter identification in dynamic systems, Journal of Engineering Mechanics (ASCE) 120 (2), (1994) 368-390.

[14] Z.H. Qureshi, T.S. Ng, G.C. Goodwin, Optimum experimental design for identification of distributed parameter systems, International Journal of Control 31 (1) (1980) 21-29.

[15] C. Papadimitriou, J.L. Beck, S.K. Au, Entropy-based optimal sensor location for structural model updating, Journal of Vibration and Control 6 (5) (2000) 781-800.

[16] J.E.T. Penny, M.I. Friswell, S.D. Garvey, The automatic choice of measurement locations for dynamic testing, in: Proceedings of the 10th International Modal Analysis Conference, San Diego, CA, 1992, pp. 30-36.

[17] D.S. Li, H.N. Li, C.P. Fritzen, The connection between effective independence and modal kinetic energy methods for sensor placement, Journal of Sound and Vibration 305 (4-5) (2007) 945-955.

[18] C. Papadimitriou, Optimal sensor placement methodology for parametric identification of structural systems, Journal of Sound and Vibration 278 (4) (2004) 923-947.

[19] C. Papadimitriou, G. Lombaert, The effect of prediction error correlation on optimal sensor placement in structural dynamics, Mechanical Systems and Signal Processing 28 (2012) 105-127.

[20] R.J. Allemang, The modal assurance criterion - twenty years of use and abuse, Sound and Vibration Magazine 37 (8) (2003) 14-23.

[21] K.V. Yuen, S.C. Kuok, Efficient Bayesian sensor placement algorithm for structural identification: a general approach for multi-type sensory systems, Earthquake Engineering \& Structural Dynamics 44 (5) (2015) 757-774.

[22] S. Joshi, S. Boyd, Sensor selection via convex optimization, IEEE Transactions on Signal Processing 57 (2) (2009) $451-462$.

[23] L. Yao, W.A. Sethares, D.C. Kammer, Sensor placement for on-orbit modal identification via a genetic algorithm, AIAA Journal 31 (10) (1993) 1922-1928.

[24] M.M. Abdullah, A. Richardson, J. Hanif, Placement of sensors/actuators on civil structures using genetic algorithms, Earthquake Engineering and Structural Dynamics 30 (8) (2001) 1167-1184

[25] TH Yi, HN Li, XD Zhang, Sensor placement on Canton Tower for health monitoring using asynchronous-climb monkey algorithm. Smart Materials and Structures 21 (12) (2012) 1-12.

[26] H. Van der Auweraer, W. Leurs, P. Mas, L. Hermans, Modal parameter estimation from inconsistent data sets, in: Proceedings of the 18th International Modal Analysis Conference, San Antonio, TX, 2000, pp. 763-771.

[27] E. Parloo, Application of frequency-domain system identification techniques in the field of operational modal analysis, PhD thesis, Vrije Universiteit Brussel, Belgium, 2003.

[28] D. Dooms, G. De Roeck, G. Degrande, G. Lombaert, M. Schevenels, S. François, StaBIL: A finite element toolbox for MATLAB, Technical Report BWM-2009-20, Department of Civil Engineering, KU Leuven (October 2009).

29] D.J. Mead, The forced vibration of one-dimensional multi-coupled periodic structures: An application to finite element analysis, Journal of Sound and vibration 319 (1-2) (2009) 282-304.

[30] E. Reynders, M. Schevenels, and G. De Roeck, MACEC 3.3: a Matlab toolbox for experimental and operational modal analysis, Report BWM-2014-06, July 2014. 
[31] E. Reynders and G. De Roeck, Reference-based combined deterministic-stochastic subspace identification for experimental and operational modal analysis, Mechanical Systems and Signal Processing 22(3) (2008) 617-637. 